\title{
STABILITY OF THE MUSCL SCHEMES FOR THE EULER EQUATIONS*
}

\author{
CHRISTOPHE BERTHON ${ }^{\dagger}$
}

\begin{abstract}
The second-order Van-Leer MUSCL schemes are actually one of the most popular high order schemes for fluid dynamic computations. In the frame work of the Euler equations, we introduce a new slope limitation procedure to enforce the scheme to preserve the invariant region: namely the positiveness of both density and pressure as soon as the associated first order scheme does it. In addition, we obtain a second-order minimum principle on the specific entropy and secondorder entropy inequalities. This new limitation is developed in the general framework of the MUSCL schemes and the choice of the numerical flux functions remains free. The proposed slope limitation can be applied to any change of variables and we do not impose the use of conservative variables in the piecewise linear reconstruction. Several examples are given in the framework of the primitive variables. Numerical 1D and 2D results are performed using several finite volume methods.
\end{abstract}

Key words. Euler equations, MUSCL schemes, invariant regions, minimum principle on the specific entropy, entropy inequalities

AMS subject classifications. 65M06 76N99 35L65

\section{Introduction}

The present work is devoted to the numerical approximations of weak solutions of the Euler equations:

$$
\left\{\begin{array}{l}
\partial_{t} \rho+\partial_{x} \rho u=0 \\
\partial_{t} \rho u+\partial_{x}\left(\rho u^{2}+p\right)=0 \\
\partial_{t} E+\partial_{x}(E+p) u=0
\end{array}\right.
$$

where the pressure is given by the perfect gas law:

$$
p=(\gamma-1)\left(E-\rho \frac{u^{2}}{2}\right), \quad \gamma \in(1,3] .
$$

For the sake of simplicity in the notations, it will be convenient to rewrite the system (1.1) as follows:

$$
\partial_{t} \mathbf{W}+\partial_{x} \mathbf{f}(\mathbf{W})=0
$$

with the state vector $\mathbf{W}: I R \times I R^{+} \rightarrow \Omega$ and the flux function $\mathbf{f}(\mathbf{W}): \Omega \rightarrow I R^{3}$ where the set $\Omega$ of the admissible states is defined by:

$$
\Omega=\left\{\mathbf{W} \in I R^{3} ; \rho>0, u \in I R, e(\mathbf{W})=E-\rho \frac{u^{2}}{2}>0\right\} .
$$

Because of the shock waves, the system (1.1) must be supplemented by an entropy inequality (see Lax [17] but also Godlewsky-Raviart [12] or Toro [26] to further details):

$$
\partial_{t} \rho \mathcal{F}(\ln s)+\partial_{x} \rho \mathcal{F}(\ln s) u \leq 0, \quad s:=s(\mathbf{W})=\frac{p}{\rho^{\gamma}}
$$

*Received: January 25, 2005; accepted (in revised version): March 23, 2005. Communicated by Tao Tang.

${ }^{\dagger}$ MAB, UMR 5466, LRC M03, Université Bordeaux I, 351 cours de la libération, 33400 Talence, France, and INRIA Futurs, projet ScAlApplix, Domaine de Voluceau-Rocquencourt, B.P. 105, 78153 Le Chesnay Cedex, France. 
where the function $\mathbf{W} \rightarrow \rho \mathcal{F}(\ln s)$ is assumed to be convex. It is well known that such a convex property is satisfied (see Godlewsky-Raviart [12], Tadmor [24]) as soon as the function $\mathcal{F}$ verifies:

$$
\mathcal{F}^{\prime}(y)<0 \quad \text { and } \quad \frac{\mathcal{F}^{\prime \prime}(y)}{\mathcal{F}^{\prime}(y)}<\frac{1}{\gamma}, \quad \forall y \in I R
$$

To simplify the notations, we introduce

$$
\overline{\mathcal{F}}(y)=\mathcal{F}(\ln y) .
$$

By definition of $\mathcal{F}$, let us note that $\overline{\mathcal{F}}$ is a decreasing function for all $y>0$.

In addition, as proved by Tadmor [24], the specific entropy $s$ satisfies the following minimum principle:

$$
s(x, t+h) \geq \min \left\{s(y, t) ;|y-x| \leq\|u\|_{\infty} h\right\} .
$$

The most usual and basic approach to approximate the solutions of (1.1) is based on a piecewise constant approximation at the time $t^{n}$ :

$$
\mathbf{W}^{n}(x)=\mathbf{W}_{i}^{n}, \quad x \in\left(x_{i-\frac{1}{2}} x_{i+\frac{1}{2}}\right),
$$

where $\left(x_{i}\right)_{i \in Z Z}$ denotes the mesh nodes. We have set $x_{i+\frac{1}{2}}=x_{i}+\left(x_{i+1}-x_{i}\right) / 2$. For the sake of simplicity in the present work, we will assume that the mesh is uniform with the size $\Delta x$. All the results stated in this paper easily extend to non-uniform grids.

The sequence $\left(\mathbf{W}_{i}^{n}\right)_{i \in Z Z}$ is defined by the following conservative scheme (see Harten-Lax-Van Leer[14], Godlewsky-Raviart [12], Toro [26], LeVeque [20]):

$$
\mathbf{W}_{i}^{n+1}=\mathbf{W}_{i}^{n}-\frac{\Delta t}{\Delta x}\left(\mathbf{F}\left(\mathbf{W}_{i}^{n}, \mathbf{W}_{i+1}^{n}\right)-\mathbf{F}\left(\mathbf{W}_{i-1}^{n}, \mathbf{W}_{i}^{n}\right)\right),
$$

where the function $\Omega \times \Omega \rightarrow \mathbf{F}\left(\mathbf{W}_{L}, \mathbf{W}_{R}\right)$ denotes the Lipschitz consistent numerical flux function (see $[14,12,20,26]$ ). The time step $\Delta t$ is assumed to satisfy the CFL like condition:

$$
\frac{\Delta t}{\Delta x} \max _{i \in Z Z}\left(\left|\lambda_{i+\frac{1}{2}}^{-}\right|,\left|\lambda_{i+\frac{1}{2}}^{+}\right|\right) \leq \frac{1}{2},
$$

where $\lambda_{i+\frac{1}{2}}^{ \pm}$are the numerical acoustic waves associated with the numerical flux function under consideration. The reader is refereed to $[12,20,26]$ to complementary details about the finite volume methods for hyperbolic system of conservation laws.

Additional stability properties can be proved for several schemes. The wellknown Godunov scheme [13, 20], but also the Lax-Friedrichs scheme (see Lax [18] or Tadmor [24] but also $[20,26]$ ), the kinetic scheme as proposed by Perthame (see Khobalatte-Perthame [16], Perthame [21] and Perthame-Qiu [23]) or the relaxation schemes (Bouchut [5], Coquel-Perthame [10], Jin-Xin [27], Berthon [4] for instance), satisfy the positiveness of the density and the internal energy, a set of discrete entropy inequalities (1.5) and a discrete formulation of the minimum principle on the specific entropy (1.7). In the sequel, the scheme (1.8) will be assumed to satisfy the following properties:

$\mathrm{P} 1: \quad \rho_{i}^{n+1}>0$ and $e_{i}^{n+1}>0$ whenever $\rho_{i}^{n}>0$ and $e_{i}^{n}>0$. 
P2: The conservative discrete entropy inequalities

$$
\begin{aligned}
\rho_{i}^{n+1} \overline{\mathcal{F}}\left(s_{i}^{n+1}\right)-\rho_{i}^{n} \overline{\mathcal{F}}\left(s_{i}^{n}\right)+ \\
\frac{\Delta t}{\Delta x}\left(\{\rho \overline{\mathcal{F}}(s) u\}\left(\mathbf{W}_{i}^{n}, \mathbf{W}_{i+1}^{n}\right)-\{\rho \overline{\mathcal{F}}(s) u\}\left(\mathbf{W}_{i-1}^{n}, \mathbf{W}_{i}^{n}\right)\right) \leq 0,
\end{aligned}
$$

for all functions $\overline{\mathcal{F}}=\mathcal{F} \circ \ln$ with $\mathcal{F}$ satisfying (1.6), where $\{\rho \overline{\mathcal{F}}(s) u\}\left(\mathbf{W}_{L}, \mathbf{W}_{R}\right)$ denotes the numerical entropy flux function.

P3: The minimum principle on the specific entropy

$$
s_{i}^{n+1} \geq \min \left(s_{i-1}^{n}, s_{i}^{n}, s_{i+1}^{n}\right) .
$$

Several strategies have been proposed to increase the accuracy of the numerical solutions. The high-order version of the first-order robust schemes is a very attractive subject. The Godunov-type scheme certainly denotes the main class of second-order scheme. It was largely studied in the last twenty years $[2,3,6,8,9,15,16,19,21,22$, 23]. Unfortunately, these procedures are, in general, based on the generalized Riemann problem (see Ben-Artzi-Falcovitz [3] or Bourgeade-LeFloch-Raviart [7]). The solutions of such problems are used with some benefits to establish stability properties like entropy inequalities (see Coquel-LeFloch [9]). The computations of these solutions turn out to be difficult and make the scheme poorly attractive. Several procedures have been proposed to approximate the Generalized Riemann Problem.

Actually, the most celebrated second-order scheme is the MUSCL scheme (see Van Leer [19]) which is systematically used in the industrial numerical simulations based on the finite volume methods. These schemes extend any first-order scheme into a second-order approximation using a very simple numerical procedure. It uses a better reconstruction than a piecewise constant function since piecewise linear functions in the form

$$
\mathbf{W}^{n}\left(x, t^{n}\right)=\mathbf{W}_{i}^{n}+\sigma_{i}^{n}\left(x-x_{i}\right), \quad x \in\left(x_{i-\frac{1}{2}}, x_{i+\frac{1}{2}}\right),
$$

are considered $[20,26]$. The value $\sigma_{i}^{n}$ denotes the slope of the linear function on the cell $\left(x_{i-\frac{1}{2}}, x_{i+\frac{1}{2}}\right)$ (see Figure 1.1). Several choices for the slope $\sigma_{i}^{n}$ are proposed in $[11,12,20,26]$. Some of these choices will be discussed latter on.

In fact, the conservative variables $\mathbf{W}$ are not the most used and several papers (see $[16,23,12])$ consider a piecewise linear reconstruction on a relevant change of variables in the form:

$$
\kappa\left(\mathbf{W}^{n}\left(x, t^{n}\right)\right)=\kappa\left(\mathbf{W}_{i}^{n}\right)+\sigma_{i}^{n}\left(x-x_{i}\right), \quad x \in\left(x_{i-\frac{1}{2}}, x_{i+\frac{1}{2}}\right),
$$

where $\kappa$ denotes a smooth change of variables. For instance, the so-called primitive variables $(\rho, u, p)$ or the variables $(\rho, u, s)$ enter this framework. For the sake of simplicity in the notations, the variables $(\rho, u, s)$ will be denoted entropic variables in the present work.

We consider the inner approximation in the cell $i$ (see Figure 1.1) located at $x=x_{i-\frac{1}{2}}$ and $x=x_{i+\frac{1}{2}}$. These approximations are denoted $\mathbf{W}_{i}^{n, \pm}$ and are defined by

$$
\mathbf{W}_{i}^{n, \pm}=\mathbf{W}^{n}\left(x_{i \pm \frac{1}{2}}, t^{n}\right)
$$

For the sake of simplicity in the remainder of the paper, we set

$$
\mathbf{W}_{i}^{n, \pm}=\mathbf{W}_{i}^{n}+\Delta \mathbf{W}_{i}^{n, \pm}
$$


where the increment $\Delta \mathbf{W}_{i}^{n, \pm}$ is defined by

$$
\Delta \mathbf{W}_{i}^{n, \pm}= \pm \frac{\Delta x}{2} \sigma_{i}^{n}
$$

in the case of linear function based on the conservative variables (1.12). If we consider linear functions based on a change of variables (1.13), $\Delta \mathbf{W}_{i}^{n, \pm}$ finds the following definition:

$$
\Delta \mathbf{W}_{i}^{n, \pm}=\kappa^{-1}\left(\kappa\left(\mathbf{W}_{i}^{n}\right) \pm \frac{\Delta x}{2} \sigma_{i}^{n}\right)-\mathbf{W}_{i}^{n}
$$

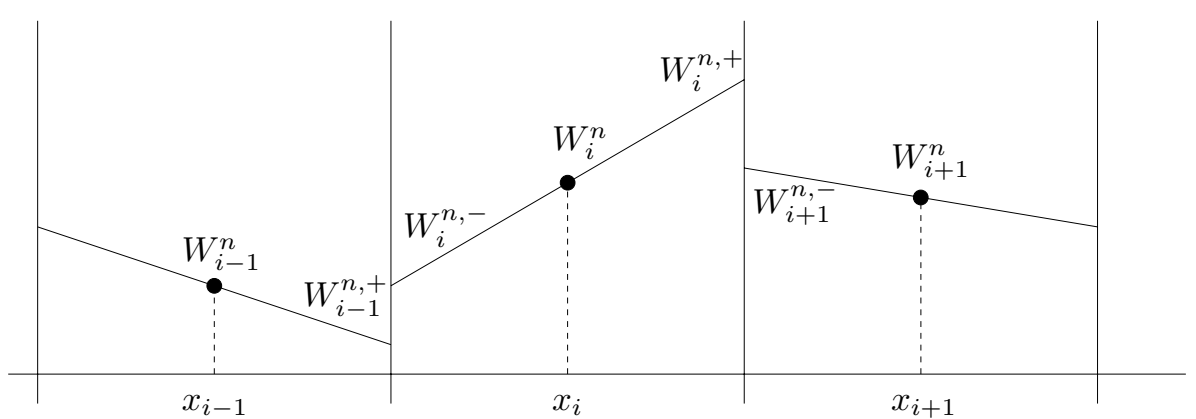

FIG. 1.1. Piece-wise linear MUSCL reconstruction

In the sequel, we will denote conservative slope with the following case:

$$
\mathbf{W}_{i}^{n,-}+\mathbf{W}_{i}^{n,+}=2 \mathbf{W}_{i}^{n}
$$

or equivalently $\Delta \mathbf{W}_{i}^{n,-}+\Delta \mathbf{W}_{i}^{n,+}=0$, while the general slope will be denoted by

$$
\mathbf{W}_{i}^{n,-}+\mathbf{W}_{i}^{n,+} \neq 2 \mathbf{W}_{i}^{n}
$$

or equivalently $\Delta \mathbf{W}_{i}^{n,-}+\Delta \mathbf{W}_{i}^{n,+} \neq 0$. Both situations will be considered in the present paper. In general, works devoted to stability properties of the MUSCL schemes (see Perthame [22] or Khobalatte-Perthame [16] for instance) solely consider the conservative slope (1.15).

The space second-order scheme writes:

$$
\mathbf{W}_{i}^{n+1}=\mathbf{W}_{i}^{n}-\frac{\Delta t}{\Delta x}\left(\mathbf{F}\left(\mathbf{W}_{i}^{n,+}, \mathbf{W}_{i+1}^{n,-}\right)-\mathbf{F}\left(\mathbf{W}_{i-1}^{n,+}, \mathbf{W}_{i}^{n,-}\right)\right),
$$

where $\mathbf{F}$ is the associated first-order flux function introduced in (1.8).

The main difficulty lies on the construction of the vector increment $\Delta \mathbf{W}_{i}^{n, \pm}$. A large literature is devoted to this subject but essentially for the scalar conservation laws and it is based on the Total Variation Diminishing criterion [9]. As emphasized by Coquel-LeFloch [9], the total variation of a solution of (1.1), in general, is not a diminishing function of time. Thus, it is necessary to focus on properties (1.5) and (1.7).

In the framework of the Euler equations (1.1), the MUSCL scheme (1.14)-(1.17) is used but, in general, the stability properties are not ensured. However, KhobalattePerthame [16] and Perthame-Qiu [23] exhibit (conservative) slope limitations in order 
to preserve the invariant region; namely the positiveness of both density and internal energy. This result is established in the framework of the kinetic scheme with a relevant like CFL restriction. In Khobalatte-Perthame [16] a new limitation based on the specific entropy is proposed to conjecture a discrete entropy minimum principle (1.11). In fact, it will be seen that this scheme enters the general approach developed in the present work (see section 2.2 devoted to the conservative slope).

The scope of this paper is to extend the properties P1, P2 and P3 to the secondorder MUSCL schemes. Arguing a relevant CFL like condition, in the next section, we develop an easy slope limitation to preserve the invariant region by the MUSCL scheme (1.14)-(1.17). In addition, we establish second-order entropy inequalities (1.10) and a second-order entropy minimum principle. These results are proved in the general context of slope limitations (1.16) and next in the specific case of conservative slope (1.15). In fact, our limitation will be understood as a correction of the usual limitation functions. Concerning the choice of the numerical flux function, we just assume that it satisfies the first-order stability properties P1, P2 and P3. The presentation of the stable limiters is concluded by the introduction of an hybrid approach. This hybrid procedure will be seen less restrictive concerning the slope limitation but for a more severe CFL restriction. Moreover, some remarks are given concerning the second-order time accuracy. The third section is devoted to the slope limitation itself. More precisely, we detail the procedure applied to the slope to enforce the stability properties. Several point of view are considered: primitive variables, entropic variables but also conservative variables. The hybrid procedure is detailed. In the last section, we illustrate what is interesting about the method. Several numerical tests are performed in 1D and 2D and comparisons with the usual MUSCL schemes are proposed. We conclude the paper with a summary and some remarks.

\section{Stability properties for second-order schemes}

In the present section, we develop a general approach to ensure the main stability properties of the MUSCL scheme (1.14)-(1.17). Actually, we do not specify the choice of the vector increment $\Delta \mathbf{W}_{i}^{n, \pm}$ which will be done in the next section. This section is solely devoted to a space limitation such that if $\Delta \mathbf{W}_{i}^{n, \pm}$ satisfies these limitations then the stability properties are enforced. Put in other words, we do not impose a piecewise linear reconstruction on the conservative variables $\mathbf{W}$ and any change of variables turns out to be admissible, primitive variables for instance, for the linear reconstruction. We conclude this section with a time second-order accurate scheme which preserves the stability properties.

2.1. The general slope limitations. Our approach is entirely based on the following remark: The MUSCL scheme (1.17) can be understood as the average over the cell $i$ of three values obtained by the first-order scheme. Indeed, let us fix a cell $i$ and a set of positive coefficients $\left(\alpha_{i}^{-}, \alpha_{i}^{\star}, \alpha_{i}^{+}\right)$such that

$$
\alpha_{i}^{-}+\alpha_{i}^{\star}+\alpha_{i}^{+}=1
$$

We introduce an intermediate state $\mathbf{W}_{i}^{n, \star}$ uniquely defined as follows:

$$
\alpha_{i}^{-} \mathbf{W}_{i}^{n,-}+\alpha_{i}^{\star} \mathbf{W}_{i}^{n, \star}+\alpha_{i}^{+} \mathbf{W}_{i}^{n,+}=\mathbf{W}_{i}^{n} \text {. }
$$

The role played by the three states $\mathbf{W}_{i}^{n,-}, \mathbf{W}_{i}^{n, \star}$ and $\mathbf{W}_{i}^{n,+}$ is displayed in the figure 2.1. Now, we propose to evolve each state with the first-order scheme (1.8) as 
follows:

$$
\begin{aligned}
& \mathbf{W}_{i}^{n+1,-}=\mathbf{W}_{i}^{n,-}-\frac{\Delta t}{\alpha_{i}^{-} \Delta x}\left(\mathbf{F}\left(\mathbf{W}_{i}^{n,-}, \mathbf{W}_{i}^{n, \star}\right)-\mathbf{F}\left(\mathbf{W}_{i-1}^{n,+}, \mathbf{W}_{i}^{n,-}\right)\right), \\
& \mathbf{W}_{i}^{n+1, \star}=\mathbf{W}_{i}^{n, \star}-\frac{\Delta t}{\alpha_{i}^{\star} \Delta x}\left(\mathbf{F}\left(\mathbf{W}_{i}^{n, \star}, \mathbf{W}_{i}^{n,+}\right)-\mathbf{F}\left(\mathbf{W}_{i}^{n,-}, \mathbf{W}_{i}^{n, \star}\right)\right), \\
& \mathbf{W}_{i}^{n+1,+}=\mathbf{W}_{i}^{n,+}-\frac{\Delta t}{\alpha_{i}^{+} \Delta x}\left(\mathbf{F}\left(\mathbf{W}_{i}^{n,+}, \mathbf{W}_{i+1}^{n,-}\right)-\mathbf{F}\left(\mathbf{W}_{i}^{n, \star}, \mathbf{W}_{i}^{n,+}\right)\right) .
\end{aligned}
$$

We immediately deduce that the updated solution by the MUSCL scheme (1.14)-(1.17) is nothing but the average of the three above updated states:

$$
\mathbf{W}_{i}^{n+1}=\alpha_{i}^{-} \mathbf{W}_{i}^{n+1,-}+\alpha_{i}^{\star} \mathbf{W}_{i}^{n+1, \star}+\alpha_{i}^{+} \mathbf{W}_{i}^{n+1,+} .
$$

This new formulation of the scheme is central to establish the expected stability properties. First, we show that the scheme (1.17) preserves the invariant region as soon as the associated first order scheme does it. Indeed, assume the CFL like condition

$$
\frac{\Delta t}{\Delta x} \max _{i \in Z Z}\left(\left|\lambda_{i,-}^{ \pm}\right|,\left|\lambda_{i, \star}^{ \pm}\right|,\left|\lambda_{i,+}^{ \pm}\right|\right) \leq \frac{1}{2} \min _{i \in Z Z}\left(\alpha_{i}^{-}, \alpha_{i}^{\star}, \alpha_{i}^{+}\right),
$$

where $\lambda_{i, \pm \star}^{ \pm}$denotes the numerical acoustic waves according to the intermediate states $\mathbf{W}_{i}^{n,-}, \mathbf{W}_{i}^{n, \star}$ and $\mathbf{W}_{i}^{n,+}$. Then, the property $\mathrm{P} 1$ can be applied to each updated partial state vector $\mathbf{W}_{i}^{n+1,-}, \mathbf{W}_{i}^{n+1, \star}$ and $\mathbf{W}_{i}^{n+1,+}$. As a consequence, as soon as $\mathbf{W}_{i}^{n, \pm \star} \in \Omega$ for all $i \in Z Z$, we have $\mathbf{W}_{i}^{n+1, \pm \star} \in \Omega$. Since $\Omega$ is a convex domain, we immediately deduce that $\mathbf{W}_{i}^{n+1}$, given by (2.2), belongs to $\Omega$. We have just established,

THEOREM 2.1. Let us consider a first-order scheme which preserves the invariant region. Assume that $\mathbf{W}_{i}^{n} \in \Omega$ and $\Delta \mathbf{W}_{i}^{n, \pm}$ satisfy for all $i \in Z Z$ :

$$
\left\{\begin{array}{l}
\mathbf{W}_{i}^{n, \pm}=\mathbf{W}_{i}^{n}+\Delta \mathbf{W}_{i}^{n, \pm} \in \Omega, \\
\mathbf{W}_{i}^{n, \star}=\mathbf{W}_{i}^{n}-\frac{\alpha_{i}^{-}}{\alpha_{i}^{\star}} \Delta \mathbf{W}_{i}^{n,-}-\frac{\alpha_{i}^{+}}{\alpha_{i}^{\star}} \Delta \mathbf{W}_{i}^{n,+} \in \Omega .
\end{array}\right.
$$

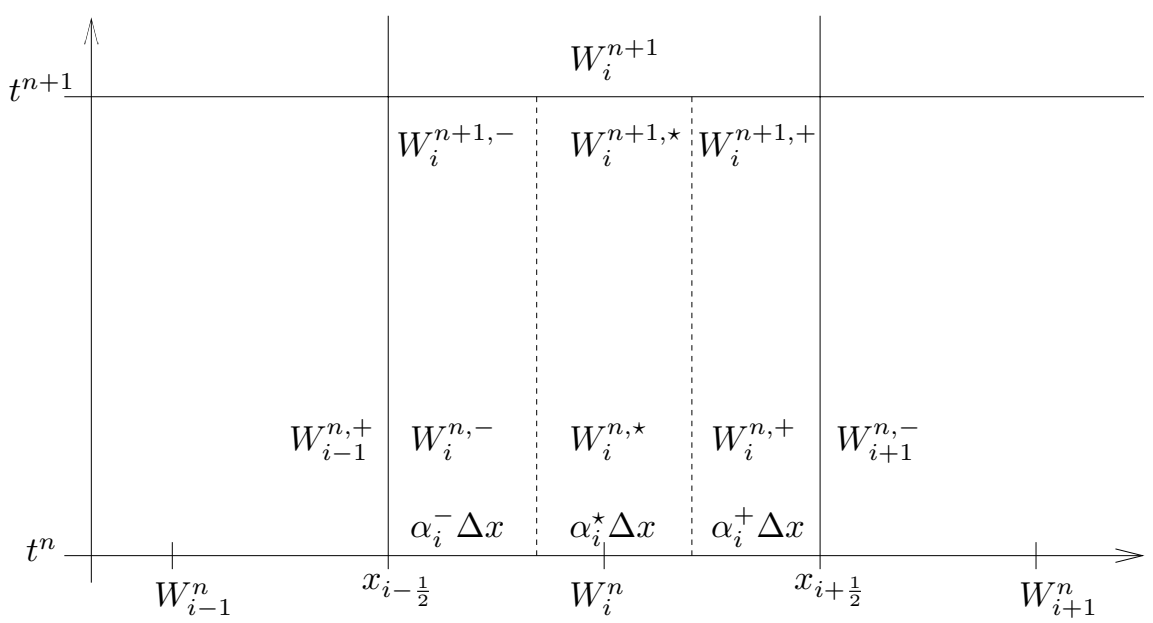

FIG. 2.1. Interpretation of the MUSCL scheme as an average of first-order scheme 
Assume the CFL restriction (2.3). Then the MUSCL scheme (1.14)-(1.17) preserves the invariant region: $\rho_{i}^{n+1}>0$ and $E_{i}^{n+1}-\left((\rho u)_{i}^{n+1}\right)^{2} /\left(2 \rho_{i}^{n+1}\right)>0$.

From now on, let us note that the limitation on the inner approximation $\mathbf{W}_{i}^{n, \pm}$ is, in general, imposed. Indeed, if $\mathbf{W}_{i}^{n, \pm}$ does not belong to $\Omega$, the numerical flux function is not defined. The actual novelty lies on the limitation on the intermediate state $\mathbf{W}_{i}^{n, \star}$. In the next sections, we will see that this new limitation is not badly restrictive and does not make complex the resulting scheme.

Now, we extend the above result with a minimum principle on the specific entropy and entropy inequalities.

THEOREM 2.2. Let the first-order scheme satisfy the properties P1, P2 and P3. Assume that $\mathbf{W}_{i}^{n} \in \Omega$ and assume that $\mathbf{W}_{i}^{n, \pm \star}$ satisfies (2.4). Assume the CFL condition (2.3). Then the second-order scheme (1.14)-(1.17) satisfies the following minimum principle:

$$
s_{i}^{n+1}=s\left(\mathbf{W}_{i}^{n+1}\right) \geq \min \left(s_{i-1}^{n,+}, s_{i}^{n, \pm \star}, s_{i+1}^{n,-}\right), \quad s_{i}^{n, \pm \star}=s\left(\mathbf{W}_{i}^{n, \pm \star}\right) .
$$

In addition, the following entropy inequalities are satisfied

$$
\begin{aligned}
& \rho_{i}^{n+1} \overline{\mathcal{F}}\left(s_{i}^{n+1}\right)-\overline{\rho \overline{\mathcal{F}}(s)_{i}^{n}}{ }^{n}+ \\
& \frac{\Delta t}{\Delta x}\left(\{\rho \overline{\mathcal{F}}(s) u\}\left(\mathbf{W}_{i}^{n,+}, \mathbf{W}_{i+1}^{n,-}\right)-\{\rho \overline{\mathcal{F}}(s) u\}\left(\mathbf{W}_{i-1}^{n,+}, \mathbf{W}_{i}^{n,-}\right) \leq 0,\right.
\end{aligned}
$$

for all functions $\overline{\mathcal{F}}=\mathcal{F} \circ \ln$ with a function $\mathcal{F}$ satisfying (1.6), where $\overline{\rho \mathcal{F}(s)}_{i}^{n}$ is defined as follows:

$$
\overline{\rho \overline{\mathcal{F}}(s)}_{i}^{n}=\alpha_{i}^{-} \rho_{i}^{n,-} \overline{\mathcal{F}}\left(s_{i}^{n,-}\right)+\alpha_{i}^{\star} \rho_{i}^{n, \star} \overline{\mathcal{F}}\left(s_{i}^{n, \star}\right)+\alpha_{i}^{+} \rho_{i}^{n,+} \overline{\mathcal{F}}\left(s_{i}^{n,+}\right) .
$$

Proof. By definition of the partial updated states $\mathbf{W}_{i}^{n+1, \pm \star}$, defined by (2.1), and since the first-order scheme satisfies (1.11), we immediately deduce the following sequence of inequalities:

$$
\left\{\begin{array}{l}
s_{i}^{n+1,-} \geq \min \left(s_{i-1}^{n,+}, s_{i}^{n,-}, s_{i}^{n, \star}\right) \geq \min \left(s_{i-1}^{n,+}, s_{i}^{n, \pm \star}, s_{i+1}^{n,-}\right), \\
s_{i}^{n+1, \star} \geq \min \left(s_{i}^{n,-}, s_{i}^{n, \star}, s_{i}^{n,+}\right) \geq \min \left(s_{i-1}^{n,+}, s_{i}^{n, \pm \star}, s_{i+-}^{n,-}\right), \\
s_{i}^{n+1,+} \geq \min \left(s_{i}^{n, \star}, s_{i}^{n,+}, s_{i+1}^{n,-}\right) \geq \min \left(s_{i-1}^{n,+}, s_{i}^{n, \pm \star}, s_{i+1}^{n,-}\right) .
\end{array}\right.
$$

Now, we apply the convex property of the entropy function $\mathbf{W} \rightarrow \rho \overline{\mathcal{F}}(s)=\rho \mathcal{F}(\ln s)$ where $\mathcal{F}$ satisfies $(1.6)$, to obtain

$$
\rho_{i}^{n+1} \overline{\mathcal{F}}\left(s_{i}^{n+1}\right) \leq \alpha_{i}^{-} \rho_{i}^{n+1,-} \overline{\mathcal{F}}\left(s_{i}^{n+1,-}\right)+\alpha_{i}^{\star} \rho_{i}^{n+1, \star} \overline{\mathcal{F}}\left(s_{i}^{n+1, \star}\right)+\alpha_{i}^{+} \rho_{i}^{n+1,+} \overline{\mathcal{F}}\left(s_{i}^{n+1,+}\right) .
$$

Since $\overline{\mathcal{F}}$ is a decreasing function, we deduce from $(2.8)$ :

$$
\begin{aligned}
\rho_{i}^{n+1} \overline{\mathcal{F}}\left(s_{i}^{n+1}\right) & \leq\left(\alpha_{i}^{-} \rho_{i}^{n+1,-}+\alpha_{i}^{\star} \rho_{i}^{n+1, \star}+\alpha_{i}^{+} \rho_{i}^{n+1,+}\right) \overline{\mathcal{F}}\left(\min \left(s_{i-1}^{n}, s_{i}^{n}, s_{i+1}^{n}\right)\right), \\
& \leq \rho_{i}^{n+1} \overline{\mathcal{F}}\left(\min \left(s_{i-1}^{n,+}, s_{i}^{n, \pm \star}, s_{i+1}^{n,-}\right)\right) .
\end{aligned}
$$

Once again, arguing the fact that $\overline{\mathcal{F}}$ decreases, we immediately obtain the expected minimum principle on the specific entropy (2.5). 
Concerning the proof of the entropy inequalities (2.6), we note that entropy inequalities are satisfied by each partial state $\mathbf{W}_{i}^{n+1, \pm \star}$ :

$$
\begin{aligned}
& \rho_{i}^{n+1,-} \overline{\mathcal{F}}\left(s_{i}^{n+1,-}\right)-\rho_{i}^{n,-} \overline{\mathcal{F}}\left(s_{i}^{n,-}\right)+ \\
& \frac{\Delta t}{\alpha_{i}^{-} \Delta x}\left(\{\rho \overline{\mathcal{F}}(s) u\}\left(\mathbf{W}_{i}^{n,-}, \mathbf{W}_{i}^{n, \star}\right)-\{\rho \overline{\mathcal{F}}(s) u\}\left(\mathbf{W}_{i-1}^{n,+}, \mathbf{W}_{i}^{n,-}\right)\right) \leq 0, \\
& \rho_{i}^{n+1, \star} \overline{\mathcal{F}}\left(s_{i}^{n+1, \star}\right)-\rho_{i}^{n, \star} \overline{\mathcal{F}}\left(s_{i}^{n, \star}\right)+ \\
& \frac{\Delta t}{\alpha_{i}^{\star} \Delta x}\left(\{\rho \overline{\mathcal{F}}(s) u\}\left(\mathbf{W}_{i}^{n, \star}, \mathbf{W}_{i}^{n,+}\right)-\{\rho \overline{\mathcal{F}}(s) u\}\left(\mathbf{W}_{i}^{n,-}, \mathbf{W}_{i}^{n, \star}\right)\right) \leq 0, \\
& \rho_{i}^{n+1,+} \overline{\mathcal{F}}\left(s_{i}^{n+1,+}\right)-\rho_{i}^{n,+} \overline{\mathcal{F}}\left(s_{i}^{n,+}\right)+ \\
& \frac{\Delta t}{\alpha_{i}^{+} \Delta x}\left(\{\rho \overline{\mathcal{F}}(s) u\}\left(\mathbf{W}_{i}^{n,+}, \mathbf{W}_{i+1}^{n,-}\right)-\{\rho \overline{\mathcal{F}}(s) u\}\left(\mathbf{W}_{i}^{n, \star}, \mathbf{W}_{i}^{n,+}\right)\right) \leq 0 .
\end{aligned}
$$

Indeed, these inequalities are directly deduced from the entropy inequalities satisfied by the first-order scheme. Owing the convex property of the function $\mathbf{W} \rightarrow \rho \overline{\mathcal{F}}(s)$, the sum of the three above inequalities gives:

$$
\begin{aligned}
\rho_{i}^{n+1} \overline{\mathcal{F}}\left(s_{i}^{n+1}\right) & -\left(\alpha_{i}^{-} \rho_{i}^{n,-} \overline{\mathcal{F}}\left(s_{i}^{n,-}\right)+\alpha_{i}^{\star} \rho_{i}^{n, \star} \overline{\mathcal{F}}\left(s_{i}^{n, \star}\right)+\alpha_{i}^{+} \rho_{i}^{n,+} \overline{\mathcal{F}}\left(s_{i}^{n,+}\right)\right) \\
& +\frac{\Delta t}{\alpha_{i}^{-} \Delta x}\left(\{\rho \overline{\mathcal{F}}(s) u\}\left(\mathbf{W}_{i}^{n,+}, \mathbf{W}_{i+1}^{n,-}\right)-\{\rho \overline{\mathcal{F}}(s) u\}\left(\mathbf{W}_{i-1}^{n,+}, \mathbf{W}_{i}^{n,-}\right)\right) \leq 0,
\end{aligned}
$$

which completes the proof.

This concludes the presentation of the main properties satisfied by the MUSCL schemes (1.14)-(1.17) as long as the first-order associated scheme is stable.

2.2. The conservative slope. We propose to focus our attention on the specific case (1.15). The conservative slope enters the above approach but simplified results can be established as soon as the condition (1.15) is satisfied. Of course, this equality is satisfied as long as the conservative variables are considered for the slope reconstruction procedure:

$$
\begin{aligned}
& \Delta \mathbf{W}_{i}^{n,+}=-\Delta \mathbf{W}_{i}^{n,-}=\Delta \mathbf{W}_{i}^{n}, \\
& \mathbf{W}_{i}^{n, \pm}=\mathbf{W}_{i}^{n} \pm \Delta \mathbf{W}_{i}^{n} .
\end{aligned}
$$

However, as proved in [16], a slope reconstruction using entropic variables $(\rho, u, s)$ is also possible.

Now, we follow the idea introduced by Perthame [22]. We set

$$
\begin{aligned}
& \mathbf{W}_{i}^{n+1,-}=\mathbf{W}_{i}^{n,-}-\frac{\Delta t}{\Delta x / 2}\left(\mathbf{F}\left(\mathbf{W}_{i}^{n,-}, \mathbf{W}_{i}^{n,+}\right)-\mathbf{F}\left(\mathbf{W}_{i-1}^{n,+}, \mathbf{W}_{i}^{n,-}\right)\right), \\
& \mathbf{W}_{i}^{n+1,+}=\mathbf{W}_{i}^{n,+}-\frac{\Delta t}{\Delta x / 2}\left(\mathbf{F}\left(\mathbf{W}_{i}^{n,+}, \mathbf{W}_{i+1}^{n,-}\right)-\mathbf{F}\left(\mathbf{W}_{i}^{n,-}, \mathbf{W}_{i}^{n,+}\right)\right) .
\end{aligned}
$$

The updated solution $\mathbf{W}_{i}^{n+1}$ given by the MUSCL scheme (1.17) thus rewrites:

$$
\mathbf{W}_{i}^{n+1}=\frac{1}{2} \mathbf{W}_{i}^{n,-}+\frac{1}{2} \mathbf{W}_{i}^{n+1} .
$$

Arguing the same notations as introduced in (2.3), let us assume the following CFL like restriction:

$$
\frac{\Delta t}{\Delta x / 2} \max _{i \in Z Z}\left(\left|\lambda_{i,-}^{ \pm}\right|,\left|\lambda_{i,+}^{ \pm}\right|\right) \leq \frac{1}{2}
$$


Now, the arguments used in the general approach give similarly the following theorem:

THEOREM 2.3. Let us consider a first-order scheme which preserves the invariant region. Assume that $\mathbf{W}_{i}^{n}$ and $\mathbf{W}_{i}^{n, \pm}$ are in $\Omega$ for all $i \in Z Z$. Assume the $C F L$ condition (2.9). Then the MUSCL scheme (1.14)-(1.17) preserves the invariant region.

In addition, assume that the first-order scheme satisfies the properties P1, P2 and P3. Then the scheme (1.14)-(1.17) satisfies the minimum principle on the specific entropy (2.5) and the entropy inequalities (2.6) hold with the following definition of $\overline{\rho \overline{\mathcal{F}}(s)}{ }_{i}$ :

$$
\overline{\rho \overline{\mathcal{F}}(s)}_{i}^{n}=\alpha_{i}^{-} \rho_{i}^{n,-} \overline{\mathcal{F}}\left(s_{i}^{n,-}\right)+\alpha_{i}^{+} \rho_{i}^{n,+} \overline{\mathcal{F}}\left(s_{i}^{n,+}\right) .
$$

The second-order MUSCL kinetic scheme proposed by Khobalatte-Perthame [16] exactly enters the present framework of conservative slope.

2.3. The hybrid limitation. We propose a third approach denoted as hybrid. Indeed, in the framework of the general slope, we will prove a stability result where we solely restrict the inner approximations $\mathbf{W}_{i}^{n, \pm}$ similarly to the case of the conservative slopes. The restriction will not use the intermediate state $\mathbf{W}_{i}^{n, \star}$. In fact, the restriction will be done on the CFL condition to obtain the expected result.

This approach is based on the following result where we prescribe a specific choice of the parameters $\alpha_{i}^{-}, \alpha_{i}^{+}$and $\alpha_{i}^{\star}=1-\left(\alpha_{i}^{-}+\alpha_{i}^{+}\right)$:

LEMMA 2.4. Let $\mathbf{W}_{i}^{n}$ and $\mathbf{W}_{i}^{n, \pm}$ be in $\Omega$ for all $i \in Z Z$. Then, there exists $\alpha_{i}^{-}$and $\alpha_{i}^{+}$in $(0,1)$ such that

$$
\mathbf{W}_{i}^{n, \star}=\mathbf{W}_{i}^{n}-\frac{\alpha_{i}^{-}}{\alpha_{i}^{\star}} \Delta \mathbf{W}_{i}^{n,-}-\frac{\alpha_{i}^{+}}{\alpha_{i}^{\star}} \Delta \mathbf{W}_{i}^{n,+} \in \Omega
$$

Proof. Assume $\alpha_{i}^{-}=\alpha_{i}^{+}=0$ then we have $\mathbf{W}_{i}^{n, \star}=\mathbf{W}_{i}^{n} \in \Omega$. By a continuity argument, we immediately obtain the result.

By virtue of this lemma, we have

THEOREM 2.5. Let us consider a first-order scheme which preserves the invariant region. Assume that $\mathbf{W}_{i}^{n} \in \Omega$ and $\mathbf{W}_{i}^{n, \pm} \in \Omega$ for all $i \in Z Z$. Let the parameters $\alpha_{i}^{-}$and $\alpha_{i}^{+}$be given by the lemma 2.4. Assume the CFL condition (2.3). Then the MUSCL scheme (1.14)-(1.17) preserves the invariant region.

In addition, assume that the first-order scheme satisfies the properties P1, P2 and P3. Then the scheme (1.14)-(1.17) satisfies the minimum principle on the specific entropy (2.5) and the entropy inequalities (2.6).

Proof. Since the parameters $\alpha_{i}^{-}$and $\alpha_{i}^{+}$are given by the lemma 2.4, we immediately deduce that all the assumptions of the theorem 2.1 are satisfied and the proof is completed.

In this procedure, the slope limitation is less restrictive than for the general slope approach. Indeed, no limitations are imposed concerning the intermediate state $\mathbf{W}_{i}^{n, \star}$. However, the modified values of the parameters $\alpha_{i}^{-}$and $\alpha_{i}^{+}$may introduce a very restrictive CFL condition. Indeed, in view of the formula (2.3), the time increment $\Delta t$ decreases proportionally with the smaller coefficient $\alpha_{i}^{ \pm \star}$. 
2.4. The time discretization. Concerning the time discretization, we propose a basic method which ensures both second-order accuracy in time and stability properties. To evolve in time from the date $t^{n}$ to $t^{n}+\Delta t$, we consider the following scheme:

$$
\left\{\begin{array}{l}
\overline{\mathbf{W}}_{i}=\mathbf{W}_{i}^{n}-\frac{\Delta t_{1}}{\Delta x}\left(\mathbf{F}\left(\mathbf{W}_{i}^{n,+}, \mathbf{W}_{i+1}^{n,-}\right)-\mathbf{F}\left(\mathbf{W}_{i-1}^{n,+}, \mathbf{W}_{i}^{n,-}\right)\right), \\
\overline{\mathbf{W}}_{i}=\overline{\mathbf{W}}_{i}-\frac{\Delta t_{2}}{\Delta x}\left(\mathbf{F}\left(\overline{\mathbf{W}}_{i}^{+}, \overline{\mathbf{W}}_{i+1}^{-}\right)-\mathbf{F}\left(\overline{\mathbf{W}}_{i-1}^{+}, \overline{\mathbf{W}}_{i}^{-}\right)\right), \\
\mathbf{W}_{i}^{n+1}=\frac{2 \Delta t_{1} \Delta t_{2}}{\left(\Delta t_{1}+\Delta t_{2}\right)^{2}} \overline{\overline{\mathbf{W}}}_{i}+\left(1-\frac{2 \Delta t_{1} \Delta t_{2}}{\left(\Delta t_{1}+\Delta t_{2}\right)^{2}}\right) \mathbf{W}_{i}^{n},
\end{array}\right.
$$

where we have set

$$
\Delta t=\frac{2 \Delta t_{1} \Delta t_{2}}{\Delta t_{1}+\Delta t_{2}}
$$

The scheme (2.12) is second-order accuracy in time. We note a discrepancy with the usual approach where $\Delta t_{1}=\Delta t_{2}=\Delta t$. In the present method, the time increment $\Delta t_{1}$ is chosen in order to ensure the CFL restriction (2.3) with $\left(\mathbf{W}_{i}^{n}\right)_{i \in Z Z}$ and the second time increment $\Delta t_{2}$ must satisfy the CFL restriction (2.3) for $\left(\overline{\mathbf{W}}_{i}\right)_{i \in Z Z}$. As soon as the slope $\Delta \mathbf{W}_{i}^{n, \pm}$ and $\Delta \overline{\mathbf{W}}_{i}^{ \pm}$satisfy the limitation (2.4), we immediately deduce that $\overline{\mathbf{W}}_{i} \in \Omega$ for all $i \in Z Z$. Since $\Omega$ is convex and $\mathbf{W}_{i}^{n+1}$ is defined by a convex sum of $\mathbf{W}_{i}^{n}$ and $\overline{\mathbf{W}}_{i}$, we have $\mathbf{W}_{i}^{n+1}$ in $\Omega$. As a consequence, the time and space second-order accurate scheme (2.12) preserves the invariant region.

In the usual case $\Delta t_{1}=\Delta t_{2}=\Delta t$, let us note that the time increment $\Delta t_{2}$ does not satisfy, in general, the CFL condition (2.3) for $\left(\overline{\mathbf{W}}_{i}\right)_{i \in Z Z}$.

\section{The limitation procedure}

In this section, we propose to detail the use of our limitation (2.4). We give several examples of limitations which appear as the most frequently used. In addition, we detail an example for the conservative and the hybrid slope limitations. Let us write the condition (2.4) as follows:

$$
\left\{\begin{array}{lll}
\rho_{i}^{n,-}>0, & \rho_{i}^{n, \star}>0, & \rho_{i}^{n,+}>0 \\
p_{i}^{n,-}>0, & p_{i}^{n, \star}>0, & p_{i}^{n,+}>0
\end{array}\right.
$$

The limitations (2.4) and (3.1) are equivalent.

3.1. Primitives variables. The first variables we propose to limit are certainly the most common choice: the primitive variables. Then, we set

$$
\left\{\begin{array}{l}
\rho_{i}^{n, \pm}=\rho_{i}^{n} \pm \Delta \rho \\
u_{i}^{n, \pm}=u_{i}^{n} \pm \Delta u \\
p_{i}^{n, \pm}=p_{i}^{n} \pm \Delta p
\end{array}\right.
$$

In the present section, we propose a limitation procedure to be applied to the increments $\Delta \rho, \Delta u$ and $\Delta p$ in order to satisfy (3.1). Presently, the increments are assumed to be known and computed by usual slope limiters (see the next section to several examples: minmod, superbee ...).

Now, we show how to modify $\Delta \rho, \Delta u$ and $\Delta p$ to satisfy (3.1). The proposed procedure is not unique and a distinct approach can be developed. First, for the sake of simplicity, we fix the parameters $\alpha_{i}^{ \pm \star}$ :

$$
\alpha_{i}^{+}=\alpha_{i}^{\star}=\alpha_{i}^{+}=\frac{1}{3} \text {. }
$$


Then, we note that $\rho_{i}^{\star}=\rho_{i}^{n}>0$. The limitation on $\Delta \rho$ thus reads:

$$
\left|\frac{\Delta \rho}{\rho_{i}^{n}}\right|<1 .
$$

Concerning the limitation on $\Delta p$, we begin with the limitation on $p_{i}^{n, \pm}$ which rewrites:

$$
\left|\frac{\Delta p}{p_{i}^{n}}\right|<1
$$

We fix $\Delta \rho$ and $\Delta p$ such that (3.3) and (3.4) are satisfied.

To conclude the limitation, we have to impose the condition on $p_{i}^{n, \star}$. After a straightforward computation, we have

$$
p_{i}^{, \star}=p_{i}^{n}-(\gamma-1)\left(1+2\left(\frac{\Delta \rho}{\rho_{i}^{n}}\right)^{2}\right) \rho_{i}^{n}(\Delta u)^{2} .
$$

With a fixed $\Delta \rho$, the increment $\Delta u$ must satisfy

$$
(\Delta u)^{2}<\frac{p_{i}^{n}}{(\gamma-1) \rho_{i}^{n}\left(1+2\left(\frac{\Delta \rho}{\rho_{i}^{n}}\right)^{2}\right)}
$$

To illustrate the limitation procedure, we propose to give an example based on the minmod limiter. Let us set

$$
\begin{aligned}
\delta \rho & =\frac{1}{2} \operatorname{minmod}\left(\left(\rho_{i}^{n}-\rho_{i-1}^{n}\right),\left(\rho_{i+1}^{n}-\rho_{i}^{n}\right)\right), \\
\delta u & =\frac{1}{2} \operatorname{minmod}\left(\left(u_{i}^{n}-u_{i-1}^{n}\right),\left(u_{i+1}^{n}-u_{i}^{n}\right)\right), \\
\delta p & =\frac{1}{2} \operatorname{minmod}\left(\left(p_{i}^{n}-p_{i-1}^{n}\right),\left(p_{i+1}^{n}-p_{i}^{n}\right)\right),
\end{aligned}
$$

where the minmod function is defined as follows:

$$
\operatorname{minmod}(a, b)=\max (0, \min (a, b))+\min (0, \max (a, b)) .
$$

Instead of the minmod function, another limiter function can be used. In the next section, we will consider both minmod and superbee functions.

By solving (3.3), (3.4) and (3.5), the increments read:

$$
\begin{aligned}
& \Delta \rho=\rho_{i}^{n} \max \left(-1, \min \left(1, \frac{\delta \rho}{\rho_{i}^{n}}\right)\right), \\
& \Delta u=\operatorname{sign}(\delta u) \sqrt{\min \left((\delta u)^{2}, \frac{p_{i}^{n}}{(\gamma-1) \rho_{i}^{n}\left(1+2\left(\frac{\Delta \rho}{\rho_{i}^{n}}\right)^{2}\right)}\right)} \\
& \Delta p=p_{i}^{n} \max \left(-1, \min \left(1, \frac{\delta p}{p_{i}^{n}}\right)\right) .
\end{aligned}
$$

Such a slope reconstruction satisfies the stability condition (3.1). Moreover, it is clear that this corrected limitation does not make more complex the scheme than the original MUSCL scheme. 
3.2. Entropic variables. We propose a linear reconstruction based on the entropic variables $(\rho, u, s)$. We set

$$
\left\{\begin{array}{l}
\rho_{i}^{n, \pm}=\rho_{i}^{n} \pm \Delta \rho \\
u_{i}^{n, \pm}=u_{i}^{n} \pm \Delta u \\
s_{i}^{n, \pm}=s_{i}^{n} \pm \Delta s
\end{array}\right.
$$

with

$$
\alpha_{i}^{-}=\alpha_{i}^{\star}=\alpha_{i}^{+}=\frac{1}{3} .
$$

Once again, we assume that the increments $\Delta \rho, \Delta u$ and $\Delta s$ are initially computed by a classical slope limiter. Now, we focus on the procedure to modify these increments to satisfy the restrictions (3.1).

The condition on $\Delta \rho$ reads:

$$
\left|\frac{\Delta \rho}{\rho_{i}^{n}}\right|<1,
$$

while the condition associated to $s_{i}^{n, \pm}$ gives

$$
\left|\frac{\Delta s}{s_{i}^{n}}\right|<1 \text {. }
$$

Concerning the last condition $p_{i}^{n, \star}>0$, equivalently, we consider $s_{i}^{n, \star}>0$. After the computations, we have:

$$
\begin{aligned}
s_{i}^{n, \star}= & 3 s_{i}^{n}-\frac{\gamma-1}{\left(\rho_{i}^{n}\right)^{\gamma-1}}\left(1+2\left(\frac{\Delta \rho}{\rho_{i}^{n}}\right)^{2}\right)(\Delta u)^{2}- \\
& \left(1+\frac{\Delta \rho}{\rho_{i}^{n}}\right)^{\gamma}\left(s_{i}^{n}+\Delta s\right)-\left(1-\frac{\Delta \rho}{\rho_{i}^{n}}\right)^{\gamma}\left(s_{i}^{n}-\Delta s\right) .
\end{aligned}
$$

Then, we deduce the following inequality:

$$
\begin{aligned}
& \frac{\gamma-1}{\left(\rho_{i}^{n}\right)^{\gamma-1}}\left(1+2\left(\frac{\Delta \rho}{\rho_{i}^{n}}\right)^{2}\right)(\Delta u)^{2}< \\
& 3 s_{i}^{n}-\left(1+\frac{\Delta \rho}{\rho_{i}^{n}}\right)^{\gamma}\left(s_{i}^{n}+\Delta s\right)-\left(1-\frac{\Delta \rho}{\rho_{i}^{n}}\right)^{\gamma}\left(s_{i}^{n}-\Delta s\right) .
\end{aligned}
$$

We note that this inequality can be solved if and only if

$$
3 s_{i}^{n}-\left(1+\frac{\Delta \rho}{\rho_{i}^{n}}\right)^{\gamma}\left(s_{i}^{n}+\Delta s\right)-\left(1-\frac{\Delta \rho}{\rho_{i}^{n}}\right)^{\gamma}\left(s_{i}^{n}-\Delta s\right)>0 .
$$

To enforce $\Delta \rho$ and $\Delta s$ such that (3.10) is satisfied, we propose to choose $\Delta \rho$ such that

$$
3 s_{i}^{n}-\left(1+\frac{\Delta \rho}{\rho_{i}^{n}}\right)^{\gamma} s_{i}^{n}-\left(1-\frac{\Delta \rho}{\rho_{i}^{n}}\right)^{\gamma} s_{i}^{n}>0 .
$$

Then, with a fixed $\Delta \rho$, the increment $\Delta s$ is reduced to satisfy (3.10).

We summarize the limitation procedure as follows: 
1. The increments $\Delta \rho, \Delta u$ and $\Delta s$ are computed by an usual approach.

2. $\Delta \rho$ is reduced to satisfy (3.7) and (3.11).

3. $\Delta s$ is reduced to satisfy $(3.8)$ and $(3.10)$.

4. $\Delta u$ is reduced to satisfy (3.9).

Let us give an example once again based on the minmod function. We set

$$
\begin{aligned}
\delta \rho & =\frac{1}{2} \operatorname{minmod}\left(\left(\rho_{i}^{n}-\rho_{i-1}^{n}\right),\left(\rho_{i+1}^{n}-\rho_{i}^{n}\right)\right), \\
\delta u & =\frac{1}{2} \operatorname{minmod}\left(\left(u_{i}^{n}-u_{i-1}^{n}\right),\left(u_{i+1}^{n}-u_{i}^{n}\right)\right), \\
\delta s & =\frac{1}{2} \operatorname{minmod}\left(\left(s_{i}^{n}-s_{i-1}^{n}\right),\left(s_{i+1}^{n}-s_{i}^{n}\right)\right) .
\end{aligned}
$$

By solving (3.7) and (3.11), the value of $\Delta \rho$ is given by

$$
\Delta \rho= \begin{cases}\rho_{i}^{n} \max \left(-1, \min \left(1, \frac{\delta \rho}{\rho_{i}^{n}}\right)\right), & \text { if } \gamma \leq \frac{\ln 3}{\ln 2} . \\ \rho_{i}^{n} \max \left(\xi^{-}, \min \left(\xi^{+}, \frac{\delta \rho}{\rho_{i}^{n}}\right)\right), & \text { otherwise }\end{cases}
$$

where $\xi^{+}$, and $\xi^{-}$denote the positive, and negative, respectively, root of the equation

$$
3-(1+\xi)^{\gamma}-(1-\xi)^{\gamma}=0 .
$$

Solving (3.10) and (3.9), we obtain the following values for $\Delta s$ and $\Delta u$ :

$$
\begin{aligned}
& \Delta s= \begin{cases}s_{i}^{n} \max \left(-1, \min \left(\frac{3-\left(1+\frac{\Delta \rho}{\rho_{i}^{n}}\right)^{\gamma}-\left(1-\frac{\Delta \rho}{\rho_{i}^{n}}\right)^{\gamma}}{\left(1+\frac{\Delta \rho}{\rho_{i}^{n}}\right)^{\gamma}-\left(1-\frac{\Delta \rho}{\rho_{i}^{n}}\right)^{\gamma}}, \frac{\Delta s}{s_{i}^{n}}\right)\right), & \text { if } \Delta \rho<0 \\
s_{i}^{n} \max \left(\frac{3-\left(1+\frac{\Delta \rho}{\rho_{i}^{n}}\right)^{\gamma}-\left(1-\frac{\Delta \rho}{\rho_{i}^{n}}\right)^{\gamma}}{\left(1-\frac{\Delta \rho}{\rho_{i}^{n}}\right)^{\gamma}-\left(1+\frac{\Delta \rho}{\rho_{i}^{n}}\right)^{\gamma}}, \min \left(1, \frac{\Delta s}{s_{i}^{n}}\right)\right), & \text { if } \Delta \rho<0, \\
s_{i}^{n} \max \left(-1, \min \left(1, \frac{\Delta s}{s_{i}^{n}}\right)\right), & \text { if } \Delta \rho=0\end{cases} \\
& \Delta u=\operatorname{sign}(\delta u) \sqrt{\min \left((\delta u)^{2}, \frac{3 s_{i}^{n}-\left(1+\frac{\Delta \rho}{\rho_{i}^{n}}\right)^{\gamma}\left(s_{i}^{n}+\Delta s\right)-\left(1-\frac{\Delta \rho}{\rho_{i}^{n}}\right)^{\gamma}\left(s_{i}^{n}-\Delta s\right)}{\frac{\gamma-1}{\left(\rho_{i}^{n}\right)^{\gamma-1}}\left(1+2\left(\frac{\Delta \rho}{\rho_{i}^{n}}\right)^{2}\right)}\right)} .
\end{aligned}
$$

We do not claim that this procedure is optimum but it yields to slopes which satisfy the limitation (3.1). Moreover, the modified limitation function does not involve very complex limitation when considering the entropic variables $(\rho, u, s)$.

3.3. Conservative variables. We propose to consider the specific case of the conservative slope (1.15). The reader is referred to the work of Khobalatte-Perthame [16] where a conservative slope limitation is proposed on the basis of entropic variables. In the present work, we just consider a linear reconstruction based on the conservative variables:

$$
\left\{\begin{array}{l}
\rho_{i}^{n, \pm}=\rho_{i}^{n} \pm \Delta \rho \\
(\rho u)_{i}^{n, \pm}=(\rho u)_{i}^{n} \pm \Delta(\rho u) \\
E_{i}^{n, \pm}=E_{i}^{n} \pm \Delta E .
\end{array}\right.
$$



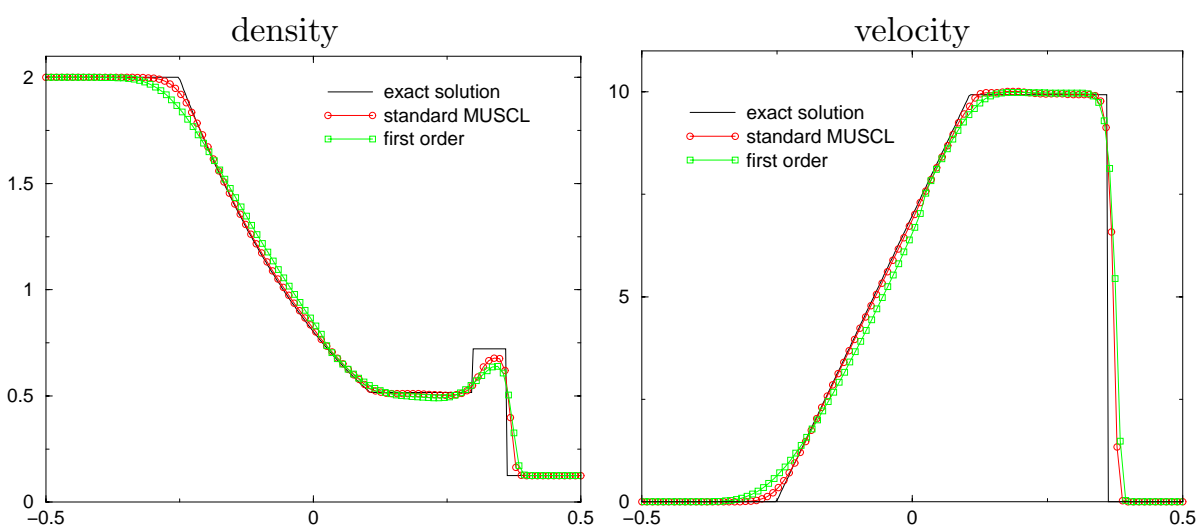

(a)
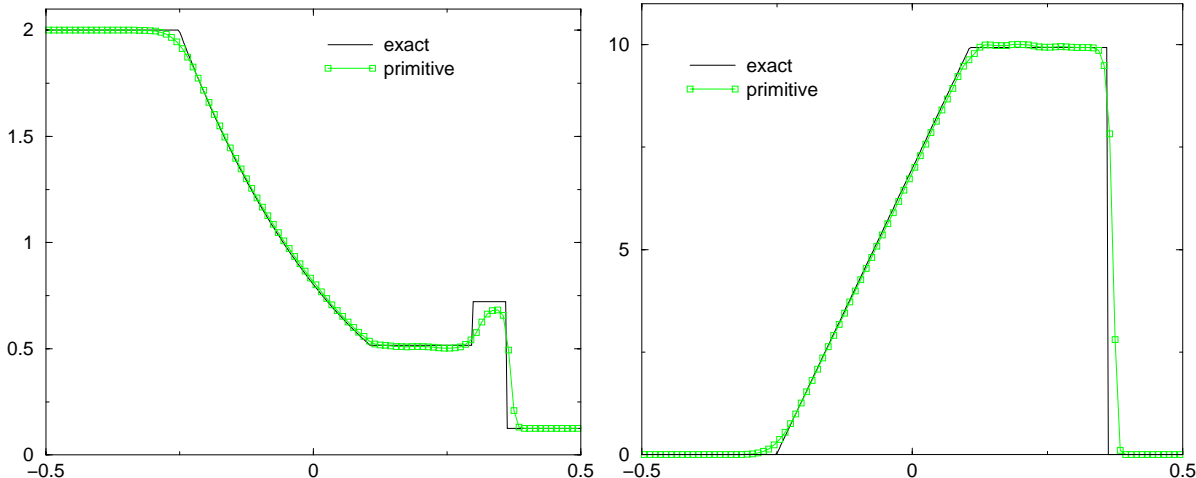

(b)
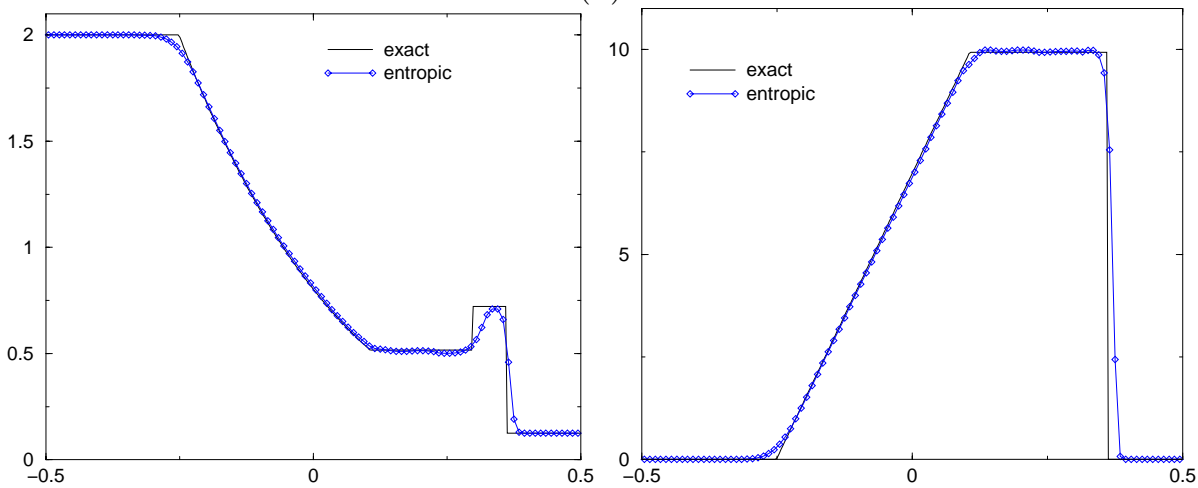

FIG. 3.1. Case 1: Relaxation MUSCL scheme, minmod slope computation: (a) modified minmod limitation for primitive variable reconstruction, (b) modified minmod limitation for entropic variables reconstruction.

Once again, the increments $\Delta \rho, \Delta(\rho u)$ and $\Delta E$ are assumed to be known. We modify these increments such that $\mathbf{W}_{i}^{n, \pm} \in \Omega$.

The condition $\rho_{i}^{n, \pm}>0$ reads:

$$
\left|\frac{\Delta \rho}{\rho_{i}^{n}}\right|<1 .
$$



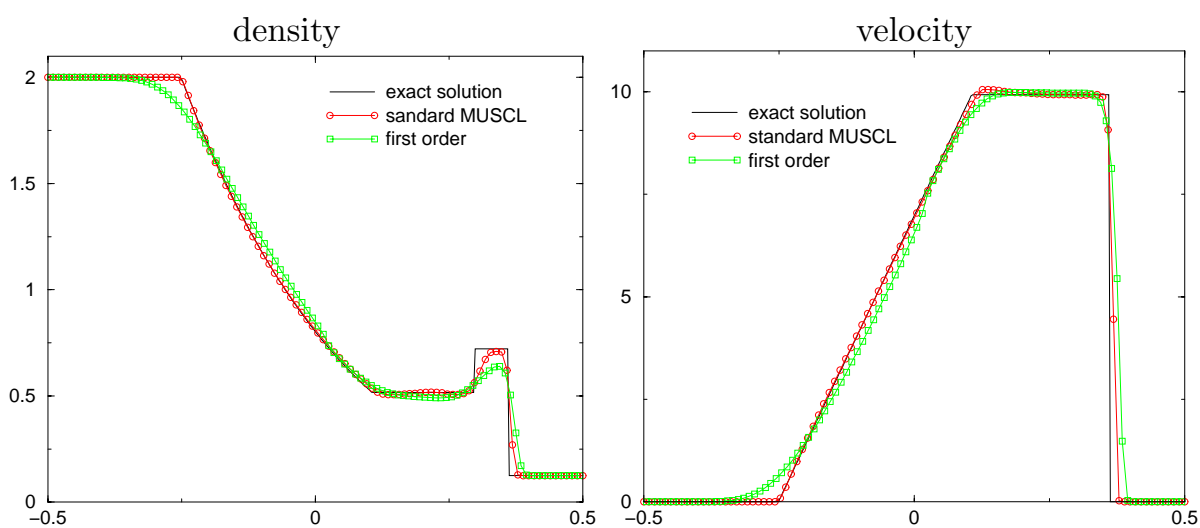

(a)
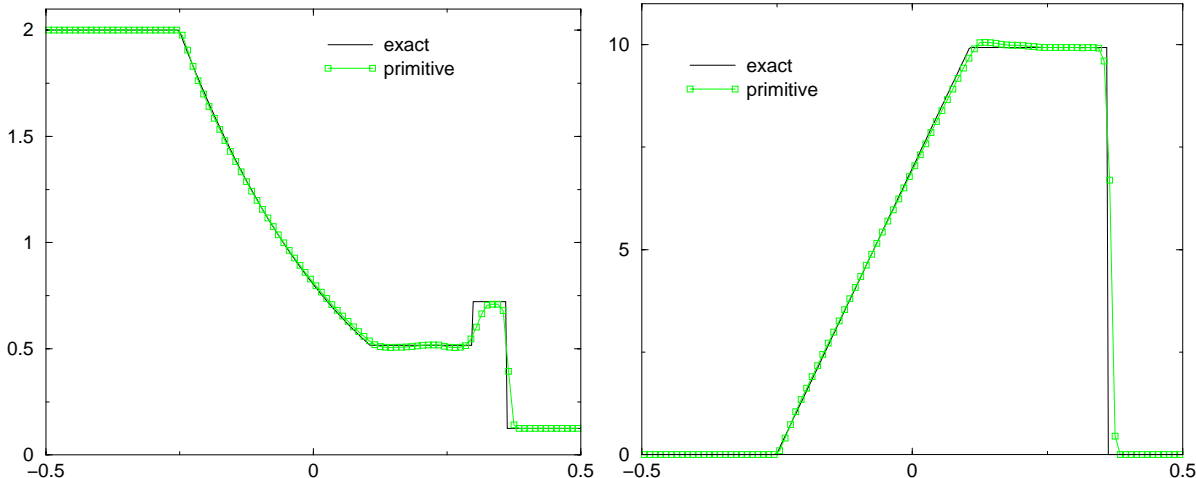

(b)
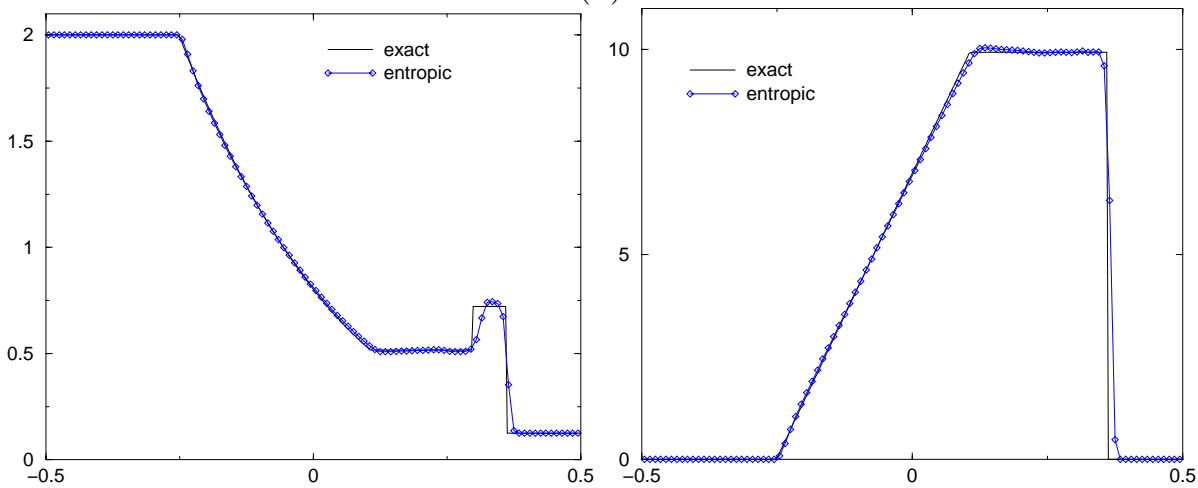

FIG. 3.2. Case 1: Relaxation MUSCL scheme, superbee slope computation: (a) modified superbee limitation for primitive variable reconstruction, (b) modified superbee limitation for entropic variable reconstruction.

The second limitation devoted to $p_{i}^{n, \pm}$ reads:

$$
E_{i}^{n, \pm}-\frac{\left((\rho u)_{i}^{n, \pm}\right)^{2}}{2 \rho_{i}^{n, \pm}}>0
$$


The sum of the inequalities (3.15) writes:

$$
2 E_{i}^{n}-\frac{1}{2}\left(\frac{\left((\rho u)_{i}^{n,-}\right)^{2}}{\rho_{i}^{n,-}}+\frac{\left((\rho u)_{i}^{n,+}\right)^{2}}{\rho_{i}^{n,+}}\right)>0 .
$$

To find $\Delta \rho$ and $\Delta(\rho u)$ satisfying (3.16), we propose to consider $\Delta \rho$ such that

$$
2 E_{i}^{n}-\frac{1}{2}\left(\frac{\left((\rho u)_{i}^{n}\right)^{2}}{\rho_{i}^{n}-\Delta \rho}+\frac{\left((\rho u)_{i}^{n}\right)^{2}}{\rho_{i}^{n}+\Delta \rho}\right)>0 .
$$

Next, for a fixed $\Delta \rho, \Delta(\rho u)$ is chosen to satisfy (3.16).

We summarize the conservative slope limitation as follows:

1. The increments $\Delta \rho, \Delta(\rho u)$ and $\Delta E$ are computed by an usual approach.

2. $\Delta \rho$ is reduced to satisfy (3.14) and (3.17).

3. $\Delta(\rho u)$ is reduced to satisfy $(3.16)$.

4. $\Delta E$ is reduced to satisfy $(3.15)$.

To propose an example of conservative slope reconstruction which satisfies (3.1), we consider (say) the minmod function and we set

$$
\begin{aligned}
& \delta \rho=\frac{1}{2} \operatorname{minmod}\left(\left(\rho_{i}^{n}-\rho_{i-1}^{n}\right),\left(\rho_{i+1}^{n}-\rho_{i}^{n}\right)\right), \\
& \delta(\rho u)=\frac{1}{2} \operatorname{minmod}\left(\left((\rho u)_{i}^{n}-(\rho u)_{i-1}^{n}\right),\left((\rho u)_{i+1}^{n}-(\rho u)_{i}^{n}\right)\right), \\
& \delta E=\frac{1}{2} \operatorname{minmod}\left(\left(E_{i}^{n}-E_{i-1}^{n}\right),\left(E_{i+1}^{n}-E_{i}^{n}\right)\right) .
\end{aligned}
$$

The following increment definition, obtained by solving (3.14)-(3.17), satisfies the stability condition (3.1):

$$
\begin{aligned}
& \Delta \rho=\rho_{i}^{n} \max \left(-\sqrt{\frac{E_{i}^{n}-\rho_{i}^{n} \frac{\left(u_{i}^{n}\right)^{2}}{2}}{E_{i}^{n}}}, \min \left(\sqrt{\frac{E_{i}^{n}-\rho_{i}^{n} \frac{\left(u_{i}^{n}\right)^{2}}{2}}{E_{i}^{n}}}, \frac{\delta \rho}{\rho_{i}^{n}}\right)\right), \\
& \Delta(\rho u)=\max \left(\xi^{-}, \min \left(\xi^{+}, \delta(\rho u)\right)\right), \\
& \Delta E=\max \left(-\left(E_{i}^{n}-\frac{\left((\rho u)_{i}^{n}+\Delta(\rho u)\right)^{2}}{2\left(\rho_{i}^{n}+\Delta \rho\right)}\right), \min \left(\left(E_{i}^{n}-\frac{\left((\rho u)_{i}^{n}-\Delta(\rho u)\right)^{2}}{2\left(\rho_{i}^{n}-\Delta \rho\right)}\right), \delta E\right)\right),
\end{aligned}
$$

where $\xi^{ \pm}$are defined as follows:

$$
\xi^{ \pm}=u_{i}^{n} \Delta \rho \pm \sqrt{2\left(\rho_{i}^{n}-\frac{(\Delta \rho)^{2}}{\rho_{i}^{n}}\right)\left(E_{i}^{n}-\rho_{i}^{n} \frac{\left(u_{i}^{n}\right)^{2}}{2}\right)} .
$$

3.4. Primitives variables for the hybrid approach. The last limitation procedure we give is devoted to the hybrid procedure. We just considered the case of primitive variables and we set

$$
\left\{\begin{array}{l}
\rho_{i}^{n, \pm}=\rho_{i}^{n} \pm \Delta \rho \\
u_{i}^{n, \pm}=u_{i}^{n} \pm \Delta u \\
p_{i}^{n, \pm}=p_{i}^{n} \pm \Delta p
\end{array}\right.
$$

where the increments are computed by an usual method. In the hybrid procedure, we have to enforce the restrictions (2.4) which rewrite in the following form:

$$
\rho_{i}^{n, \pm}>0, \quad p_{i}^{n, \pm}>0
$$


(a)

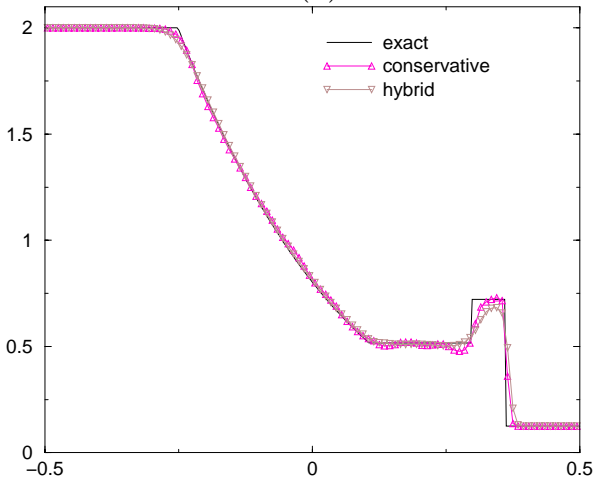

(b)

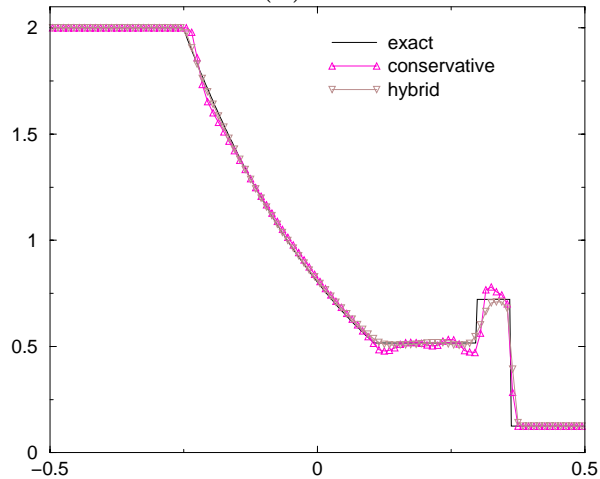

FIG. 3.3. Case 1: Relaxation MUSCL scheme (a) modified minmod slope computation for the conservative and the hybrid approaches (b) modified superbee slope computation for the conservative and the hybrid approaches.

These two conditions are satisfied as soon as the increments $\Delta \rho$ and $\Delta p$ verify the inequalities (3.3) and (3.4).

Now, the hybrid approach is imposed to satisfy the lemma 2.4. So, we are searching for parameters $\alpha_{i}^{-}$and $\alpha_{i}^{+}$such that the condition (2.11) is satisfied. For the sake of simplicity, we propose to consider parameters in the form:

$$
\alpha_{i}^{-}=\alpha_{i}^{+}=\alpha_{i} \in(0,1 / 2) .
$$

As a consequence, we obtain after computations:

$$
\begin{aligned}
& \rho_{i}^{n, \star}=\rho_{i}^{n}, \\
& p_{i}^{n, \star}=p_{i}^{n}-\frac{\alpha_{i}}{\left(1-2 \alpha_{i}\right)^{2}}(\gamma-1) \rho_{i}^{n}\left(\left(1-2 \alpha_{i}\right)+2 \alpha\left(\frac{\Delta \rho}{\rho_{i}^{n}}\right)^{2}\right)(\Delta u)^{2} .
\end{aligned}
$$

With fixed increments $\Delta \rho, \Delta u$ and $\Delta p$, there exists $\alpha_{i} \in(0,1 / 2)$ such that

$$
p_{i}^{n, \star}>0
$$

The parameter $\alpha_{i}$ is the solution of a second order inequality not detailed here.

In view of the CFL condition (2.3), the relevant value of $\alpha_{i}$ may reduce drastically the time increment $\Delta t$ (see the numerical test 2). For numerical simulations, the coefficient $\alpha_{i}$ is chosen in order to maximize the value of $\min \left(\alpha_{i}^{-}, \alpha_{i}^{\star}, \alpha_{i}^{+}\right)$; the better choice of $\alpha_{i}$ is given by $1 / 3$.

\section{Numerical results}

In this section, we illustrate our numerical procedure with several $1 \mathrm{D}$ and $2 \mathrm{D}$ tests. Concerning the 1D numerical tests, they are performed using the same strategy. The mesh is assumed to be uniform and made of 100 cells. The CFL number is fixed to 0.5 according to the CFL like restriction (2.3). With $\alpha_{i}^{ \pm \star}=1 / 3$, let us note that the CFL condition reads

$$
\frac{\Delta t}{\Delta x} \max _{i \in Z Z}\left(\left|\lambda_{i, \pm \star}^{ \pm}\right|\right) \leq \frac{1}{6} .
$$


(a)

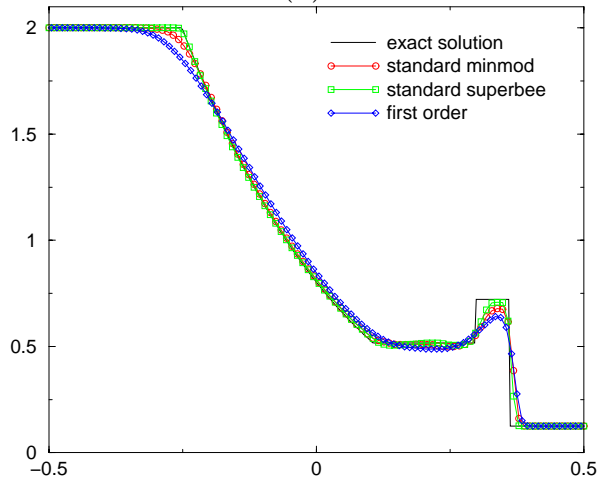

(b)

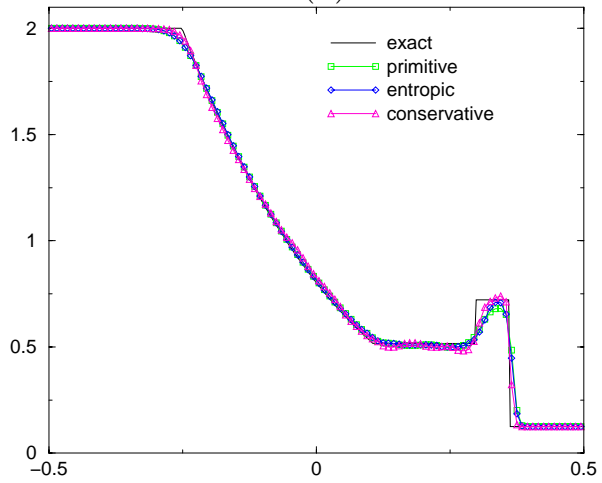

(c)

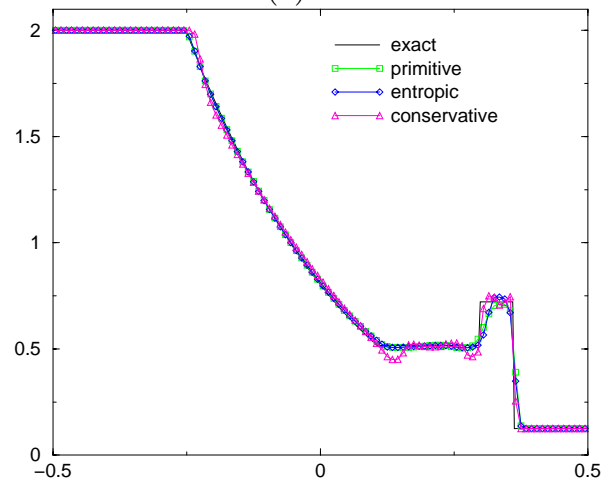

FIG. 3.4. Case 1: Kinetic MUSCL scheme (a) standard approach with a primitive variable reconstruction (b) modified minmod slope computation for the primitive, the entropic and the conservative variables (c) modified superbee slope computation for the primitive, the entropic and the conservative variables.

Two distinct first-order finite volume schemes are considered: the kinetic scheme $[16,21,22,23]$ and the Suliciu relaxation scheme $[1,4,5,10]$. Concerning the computation of the slopes, we consider two of the most popular formulas: the minmod and the superbee functions (for instance, see [11, 12, 20, 26] for further details). These limitations are thus modified according to the above theory (see section 3 and the examples therein).

The numerical results are systematically compared with the standard MUSCL approach. In the present work, the standard MUSCL scheme is given by (1.8) where the reconstruction is performed on the primitive variables $(\rho, u, p)$. Concerning the CFL condition, we adopt the following restriction:

$$
\frac{\Delta t}{\Delta x} \max _{i \in Z Z}\left(\left|\lambda_{i, \pm}^{ \pm}\right|\right) \leq \frac{1}{6}
$$

In general, the CFL number is fixed to 0.5 . To be consistent with our CFL condition (4.1), we consider a CFL number equal to $1 / 6$ for the standard MUSCL scheme.

The first test corresponds to a Riemann solution made of a shock wave and a rarefaction wave separated by a contact discontinuity. The initial data is made of two 
(a)

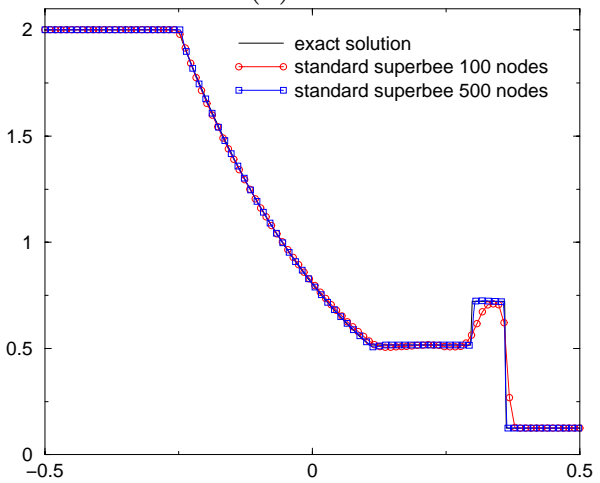

(c)

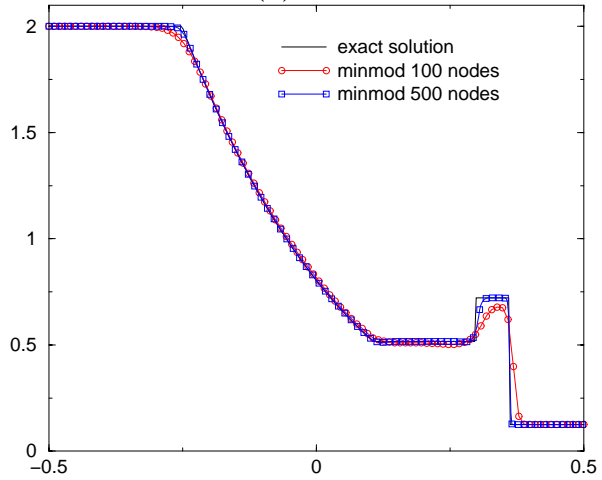

(b)

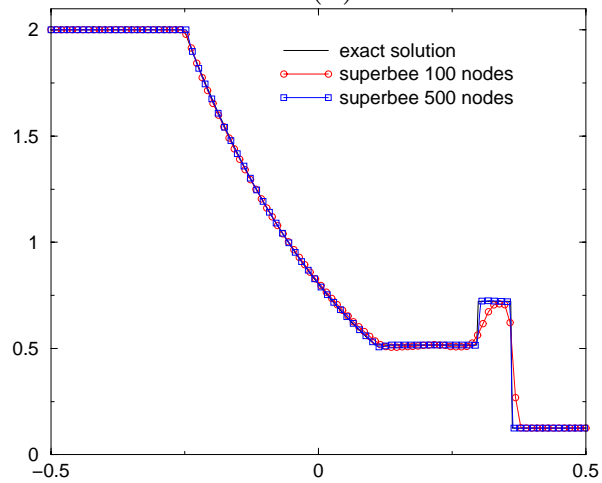

(d)

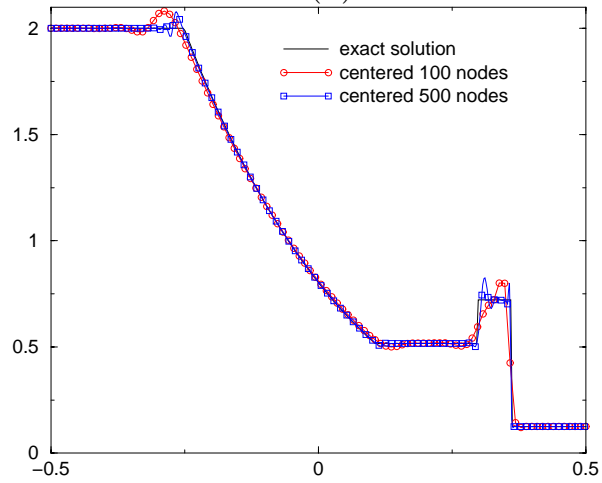

FIG. 3.5. Case 1: Relaxation MUSCL scheme with primitive variable reconstruction (a) classical superbee slope computation (b) modified superbee slope computation (c) modified minmod slope computation (d) modified centered slope computation.

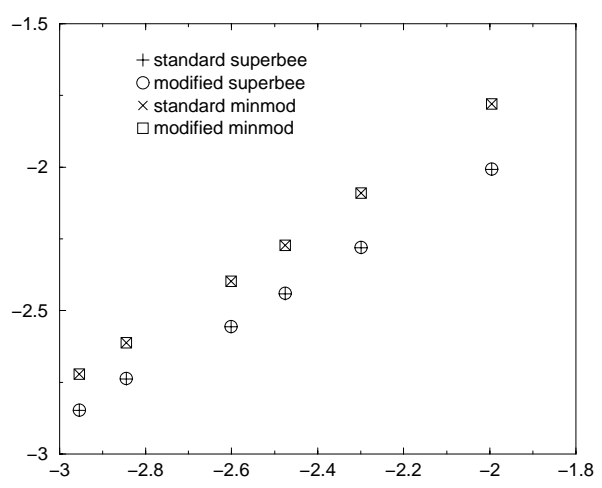

FIG. 3.6. Case 1: Logarithmic $L^{1}$-error versus $\log (\Delta x)$ for the relaxation MUSCL scheme with standard and modified limiter for the primitive variable reconstruction.

constant states defined as follows:

$$
\begin{array}{lll}
\rho_{L}=2, & u_{L}=0, & p_{L}=100 \\
\rho_{R}=0.125, & u_{R}=0, & p_{R}=0.1 .
\end{array}
$$


(a)

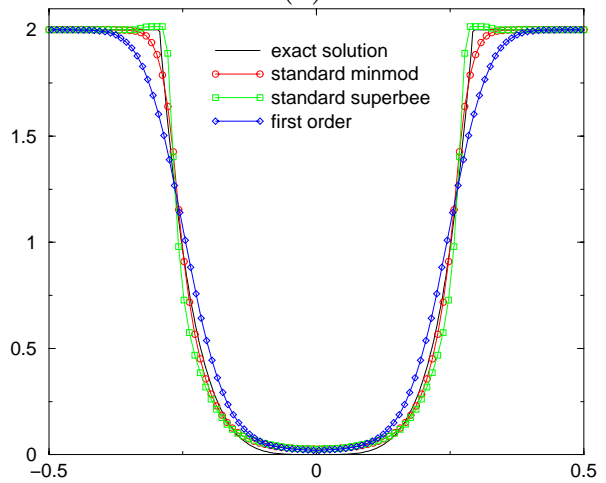

(b)

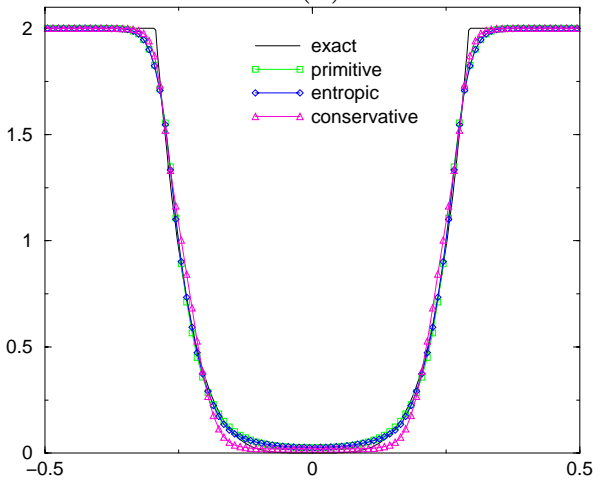

(c)

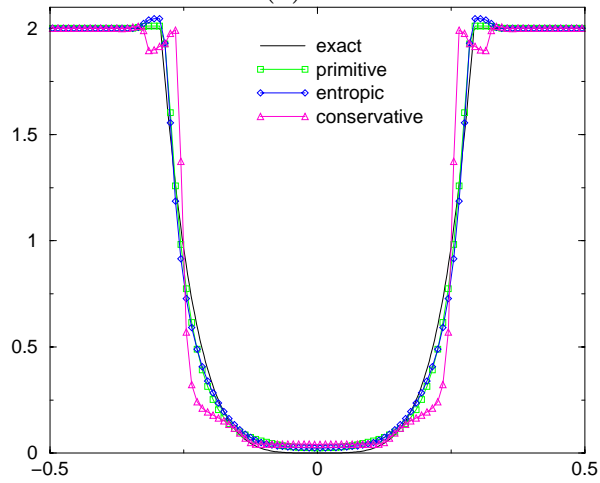

FIG. 3.7. Case 2: Relaxation MUSCL scheme (a) standard approach with a primitive variable reconstruction (b) minmod slope computation for the primitive, the entropic and the conservative variables (c) superbee slope computation for the primitive, the entropic and the conservative variables.

The left and right states are separated by a discontinuity located at $x=0$. The solution is displayed at the time $t=0.03$. The numerical results are displayed in the figures 3.1 to 3.4 .

In the figure 3.1, we present the results obtained with the Suliciu relaxation scheme. To be compared, we give the first-order accurate result and the approximate solution obtained with the classical second-order MUSCL scheme. The slope is computed with the minmod function (see [20]) and modified according to (2.4). In this figure, also we display the approximate results obtained involving the primitive variable reconstruction (section 3.1) and the entropic variable reconstruction (section 3.2 ).

In the next figure 3.2, the same methods are considered but for the modified superbee function according to the restriction (2.4). The numerical results are supplemented by approximations obtained involving conservative variables (section 3.3) and the hybrid approach (section 3.4). These results are displayed in the figure 3.3.

The figure 3.4 is devoted to the numerical results using the kinetic scheme. For the sake of clarity, we do not display the hybrid approach. To conclude the discussion about this test, we emphasize that the new limitation procedure does not provide the oscillations. The figure 3.5 illustrates this point. For two mesh refinements, we display 
(a)

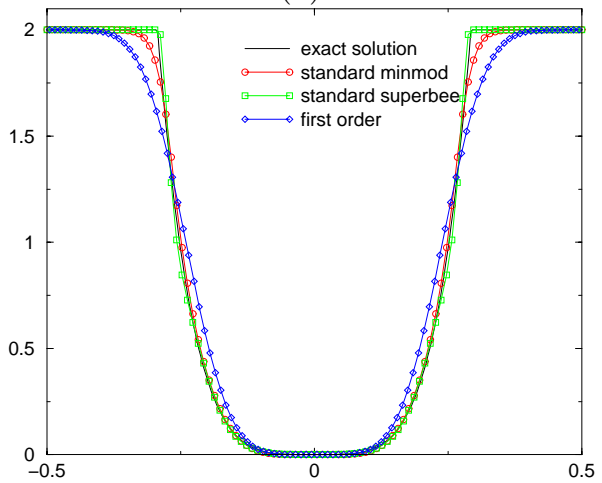

(b)

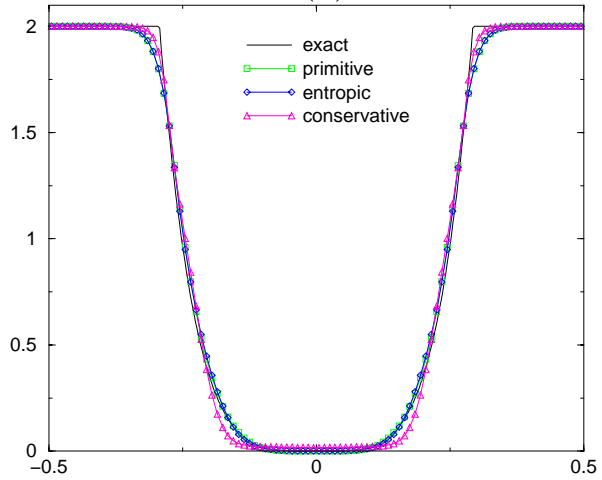

(c)

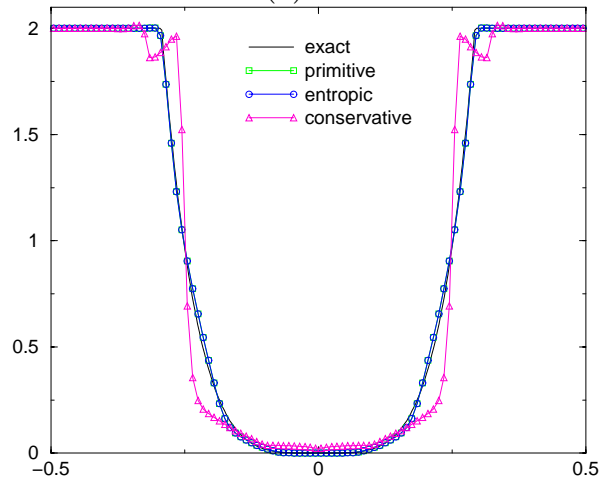

FIG. 3.8. Case 2: Kinetic MUSCL scheme (a) standard approach with a primitive variable reconstruction (b) modified minmod slope computation for the primitive, the entropic and the conservative variables (c) modified superbee slope computation for the primitive, the entropic and the conservative variables.

the result obtained with the classical superbee MUSCL scheme and with the modified superbee and modified minmod slope reconstructions. In addition, we display the result obtained with the centered slope, known to involve large oscillations. We just recall that the intermediate increment $\delta \rho, \delta u$ and $\delta p$, introduced in section 3 to modify the limitation functions, reads as follows in the case of centered slopes:

$$
\begin{aligned}
& \delta \rho=\frac{\rho_{i+1}^{n}-\rho_{i-1}^{n}}{2}, \\
& \delta u=\frac{u_{i+1}^{n}-u_{i-1}^{n}}{2}, \\
& \delta p=\frac{p_{i+1}^{n}-p_{i-1}^{n}}{2} .
\end{aligned}
$$

Two (small) oscillations persist for a fine mesh but the approximate solution turns out to be in a very good agreement with the exact solution.

To conclude the first numerical experiment, we note that the standard MUSCL approaches and the modified MUSCL reconstruction give the same level of accuracy. This remark is emphasized in the figure 3.6 where logarithmic $L^{1}$-error for both standard and modified MUSCL reconstruction. This point is crucial since it confirms that 
(a)

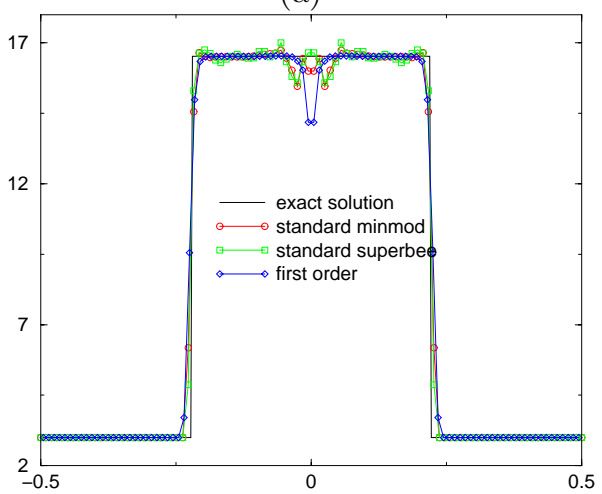

(b)

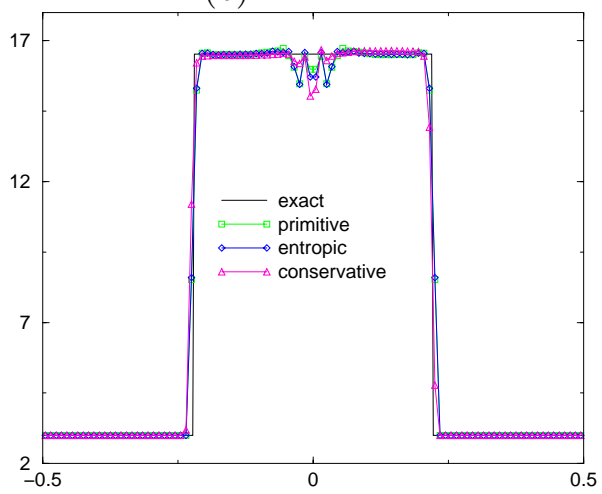

(c)

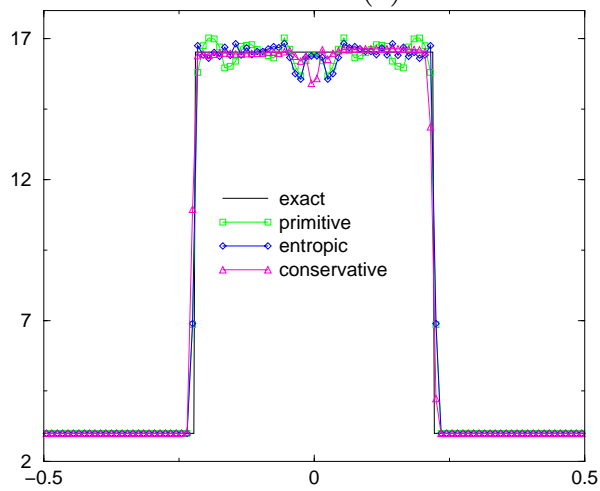

FIG. 3.9. Case 3: Relaxation MUSCL scheme (a) standard approach with a primitive variable reconstruction (b) modified minmod slope computation for the primitive, the entropic and the conservative variables (c) modified superbee slope computation for the primitive, the entropic and the conservative variables.

our limitation does not reduce the order of the numerical method.

In the second test, we consider a Riemann solution made of two rarefaction waves. The left and right states which made the initial data are defined as follows:

$$
\begin{array}{lll}
\rho_{L}=2, & u_{L}=-12, & p_{L}=10 \\
\rho_{R}=2, & u_{R}=12, & p_{R}=10 .
\end{array}
$$

The solution is displayed at the time $t=0.02$. The figure 3.7 is devoted to the numerical results performed with the relaxation scheme while in the figure 3.8 , we display the results completed with the kinetic scheme. We present the approximations based on the modified minmod and modified superbee slope computations. The results using primitive, entropic and conservative variable reconstruction are given. Let us note that the hybrid method involves a very small time increment and no results are performed by this procedure for the test of two rarefaction waves.

The last $1 \mathrm{D}$ test concerns a Riemann solution made of two shock waves. The initial data for this test is defined as follows:

$$
\begin{array}{lll}
\rho_{L}=3, & u_{L}=100, & p_{L}=573, \\
\rho_{R}=3, & u_{R}=-100, & p_{R}=573 .
\end{array}
$$


(a)

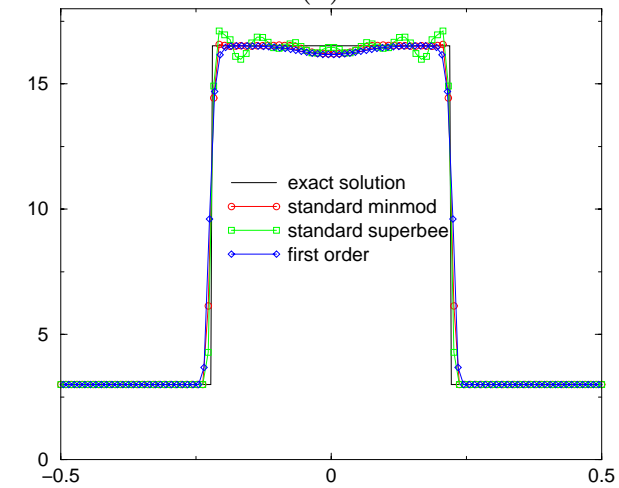

(b)

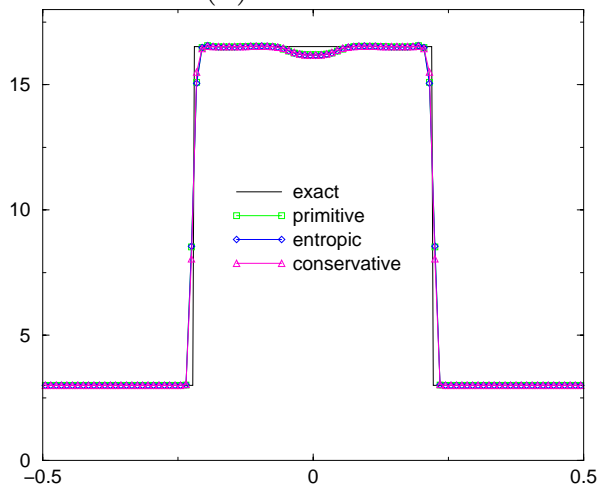

(c)

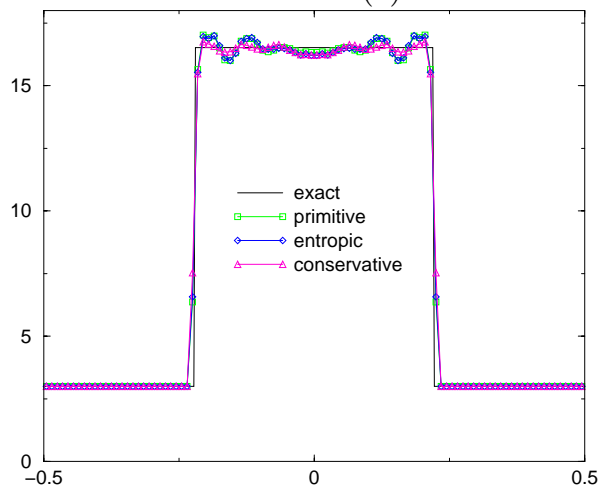

FIG. 3.10. Case 3: Kinetic MUSCL scheme (a) standard approach with a primitive variable reconstruction (b) modified minmod slope computation for the primitive, the entropic and the conservative variables (c) modified superbee slope computation for the primitive, the entropic and the conservative variables.

The numerical solutions are displayed at the time $t=0.01$ in the figures 3.9 and 3.10 . The same strategy of presentation used for the second test is adopted here. We note that the approaches based on the superbee function, standard or modified, involve oscillations and the better results are obtained with the minmod function.

In the last simulation, we propose a 2D Riemann problem (see Kurganov-Tadmor $[25])$. The initial data is defined as follows:

$$
\begin{array}{lc|ll}
\rho_{1}=0.5323 & p_{1}=0.3 & \rho_{2}=1.5 & p_{2}=1.5 \\
u_{1}=1.206 & v_{1}=0, & u_{2}=0 & v_{2}=0 \\
\hline \rho_{3}=0.138 & p_{3}=0.029 & \rho_{4}=0.5323 & p_{4}=0.3 \\
u_{3}=1.206 & v_{3}=1.206 & u_{4}=0 & v_{4}=1.206 .
\end{array}
$$

At the time $t=0.3$, the density solution is displayed in the figure 3.11 .

\section{5. conclusion}

In this paper, we propose a new version of the celebrate MUSCL scheme to approximate the solutions of the Euler equations. We focus our attention on the linear reconstruction procedure to enforce several stability properties. The obtained secondorder MUSCL scheme ensures the numerical solutions to satisfy the positiveness of 


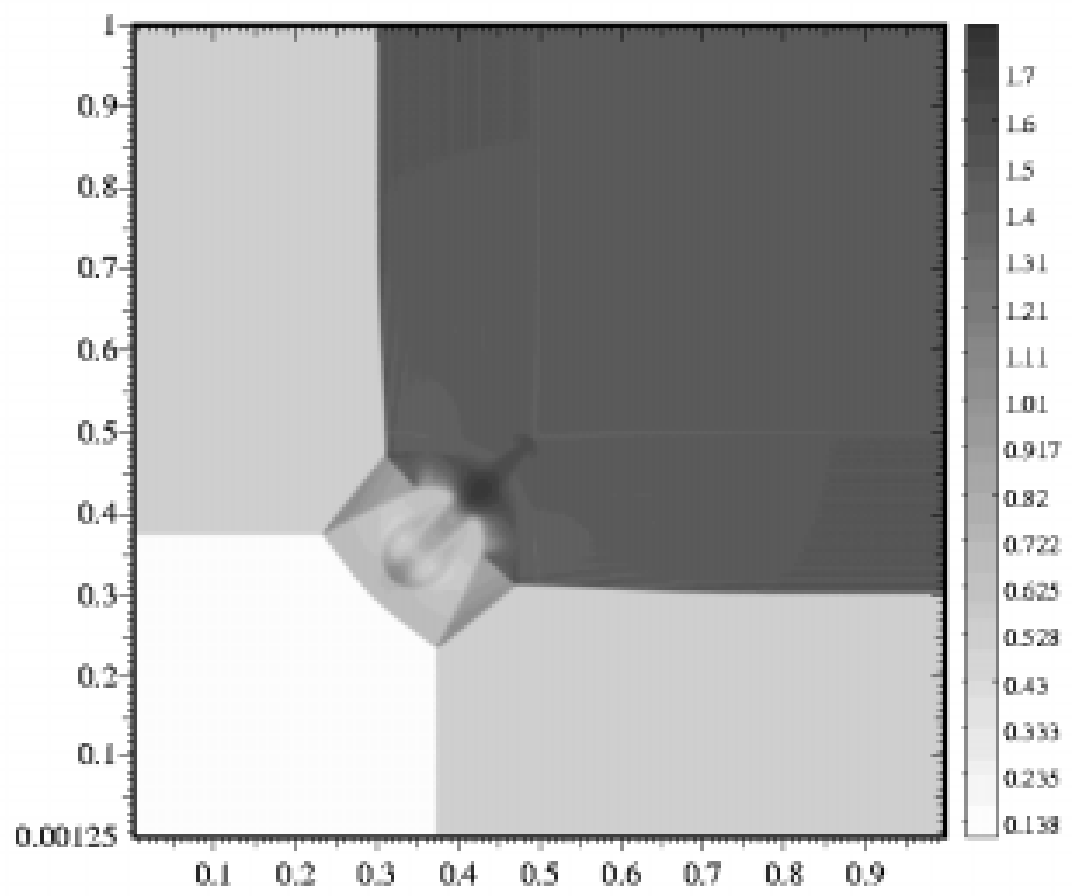

FIG. 3.11. 2D Test with a $400 x 400$ structured mesh: Relaxation MUSCL scheme using modified superbee slope function on the primitive variables.

the density and the pressure but also a second-order entropy minimum principle. In addition, second-order entropy inequalities are established. These results are obtained arguing a relevant CFL condition. We recall that no CFL condition is, actually, established concerning the Euler equations excepted in the work of Perthame et al. $[16,23]$. In general, a CFL condition in the form (4.2), with 0.5 as CFL number, is considered. As specified in [26], it should be used with caution. In the case of conservative reconstruction, the CFL restriction we find is according to several works $[16,23]$ devoted to stability of MUSCL schemes.

These stability results are obtained independently of the choice of the numerical flux function as long as several stability properties are satisfied by the numerical flux function. Put in other words, the proposed MUSCL method preserves the stability properties of the associated first-order scheme. The present MUSCL method is shown to be a simple modification of the standard approach and its implementation is obtained after few lines of code (see the examples given in section 3 ).

To illustrate the method, numerical tests are proposed. They are performed using two distinct numerical flux functions. Several slope reconstructions are detailed. These slopes are based on conservative and non-conservative variables. The results illustrate what is interesting about the method and establish that the order of accuracy of the original MUSCL scheme is preserved.

Acknowledgments. The author thank B. Nkonga and B. Dubroca for their helpful suggestions and comments. 


\section{REFERENCES}

[1] M. Baudin, C. Berthon, F. Coquel, R. Masson and H. Tran, A relaxation method for two-phase flow models with hydrodynamic closure law, Numer. Math. 99, 3, 411-440, 2005.

[2] J. B. Bell, P. Colella and J. A. Trangenstein, Higher order Godunov methods for general systems of hyperbolic conservation laws, J. Comput. Phys. 82, 2, 362-397, 1989.

[3] M. Ben-Artzi and J. Falcovitz, A second-order Godunov-type scheme for compressible fluid dynamics, J. Comput. Phys. 55, 1, 1-32, 1984.

[4] C. Berthon, Inégalités d'entropie pour un schéma de relaxation, C. R. Acad. Sci. I, Math. 340, 63-68, 2005.

[5] F. Bouchut, Nonlinear stability of finite volume methods for hyperbolic conservation laws, and well-balanced schemes for sources, Frontiers in Mathematics series, Birkhäuser, 2004.

[6] F. Bouchut, Ch. Bourdarias and B. Perthame, A MUSCL method satisfying all the numerical entropy inequalities, Math. Comp. 65, 216, 1439-1461, 1996.

[7] A. Bourgeade, P. LeFloch and P. A. Raviart, An asymptotic expansion for the solution of the generalized Riemann problem. II. Application to the equations of gas dynamics, Ann. Inst. H. Poincar Anal. Non Linaire 6, 6, 437-480, 1989.

[8] P. Colella, A direct Eulerian MUSCL scheme for gas dynamics, SIAM J. Sci. Statist. Comput. $6,1,104-117,1985$.

[9] F. Coquel and P. LeFloch, An entropy satisfying MUSCL scheme for systems of conservation laws, Numer. Math. 74, 1, 1-33, 1996.

[10] F. Coquel and B. Perthame, Relaxation of energy and approximate Riemann solvers for general pressure laws in fluid dynamics, SIAM J. Numer. Anal. 35, 6, 2223-2249, 1998.

[11] E. Godlewsky and P. A. Raviart, Hyperbolic Systems of Conservations Laws, Tome 1, SMAI , Ellipse, 1991.

[12] E. Godlewsky and P. A. Raviart, Hyperbolic Systems of Conservations Laws, Tome 2, Applied Mathematical Sciences, Springer, 118, 1995.

[13] S. K. Godunov, A difference scheme for numerical computation of discontinuous solutions of equations of fluids dynamics, Math. Sbornik, 47, 271-306, 1959.

[14] A. Harten, P. D. Lax and B. Van Leer, On upstream differencing and Godunov-type schemes for hyperbolic conservation laws, SIAM Review, 25, 1, 35-61, 1983.

[15] A. Harten and S. Osher, Uniformly high-order accurate nonoscillatory schemes, SIAM J. Numer. Anal. 24, 2, 279-309, 1987.

[16] B. Khobalatte and B. Perthame, Maximum principle on the entropy and second-order kinetic schemes, Math. of Comp., 62, 205, 119-131, 1994.

[17] P. D. Lax, Hyperbolic systems of conservation laws and the mathematical theory of shock waves, Conference Board of the Mathematical Sciences Regional Conference Series in Applied Mathematics, SIAM, Philadelphia, 11, 1973.

[18] P. D. Lax, Shock waves and entropy, Contributions to nonlinear functional analysis, E. H. Zarantonello, Ed., 603-634, 1971.

[19] B. van Leer, Towards the ultimate conservative difference scheme. $V$. A second-order sequel to Godunov's method, J. comput. Phys., 32, 101-136, 1979.

[20] R. J. LeVeque, Finite Volume Methods for Hyperbolic Problems, Cambridge Texts in Applied Mathematics, Cambridge University Press, Cambridge, 2002.

[21] B. Perthame, Boltzmann type schemes for gas dynamics and the entropy property, SIAM J. Numer. Anal., 27, 6, 1405-1421, 1990.

[22] B. Perthame, Second-order Boltzmann schemes for compressible Euler equations in one and two space dimensions, SIAM J. Numer. Anal., 29, 1, 1-19, 1992.

[23] B. Perthame and Y. Qiu, A variant of Van Leer's method for multidimensional systems of conservation laws, J. Comput. Phys., 112, 2, 370-381, 1994.

[24] E. Tadmor, A minimum entropy principle in the gas dynamics equations Appl. Numer. Math., 2, 3-5, 211-219, 1986

[25] A. Kurganov and E. Tadmor, Solution of two-dimensional Riemann problems for gas dynamics without Riemann problem solvers, Numer. Methods Partial Differential Equations, 18, 5, 584-608, 2002.

[26] E. F. Toro, Riemann Solvers and Numerical Methods for Fluid Dynamics. A Practical Introduction, Second edition, Springer-Verlag, Berlin, 1999.

[27] S. Jin and Z. Xin, The relaxation scheme for systems of conservation laws in arbitrary space dimensions, Comm. Pure and Appl. Math., 48, 235-276, 1995. 